\title{
FINITE- AND INFINITE-TIME RUIN PROBABILITIES WITH GENERAL STOCHASTIC INVESTMENT RETURN PROCESSES AND BIVARIATE UPPER TAIL INDEPENDENT AND HEAVY-TAILED CLAIMS
}

\author{
FENGLONG GUO, Nanjing Audit University, and University of Electronic Science \\ and Technology of China \\ DINGCHENG WANG, ${ }^{*}$ Nanjing Audit University, Australian National University, \\ and University of Electronic Science and Technology of China
}

\begin{abstract}
In this paper we investigate the asymptotic behaviors of the finite- and infinite-time ruin probabilities for a Poisson risk model with stochastic investment returns which constitute a general adapted càdlàg process and heavy-tailed claim sizes which are bivariate upper tail independent. The results of this paper show that the asymptotic ruin probabilities are dominated by the extreme of insurance risk but not by that of investment risk. As applications of the results, we discuss four special cases when the investment returns are determined by a fractional Brownian motion, an integrated Vasicek model, an integrated Cox-Ingersoll-Ross model, and the Heston model.

Keywords: Ruin probability; heavy tail; investment return process; upper tail dependence; fractional Brownian motion; Vasicek model; Cox-Ingersoll-Ross model; Heston model

2010 Mathematics Subject Classification: Primary 62P05
\end{abstract}

Secondary 62P20; 91B30

\section{Introduction}

Throughout this paper, let $\left(\Omega, \mathcal{F},\left(\mathcal{F}_{t}\right)_{t \geq 0}, \mathbb{P}\right)$ be a filtered complete probability space on which all stochastic quantities are defined. The filtration $\left(\mathcal{F}_{t}\right)_{t \geq 0}$ is right continuous and all stochastic processes to be defined in this paper are adapted to the filtration.

Suppose that the claim sizes $\left\{X_{n}, n \geq 1\right\}$ of an insurance business are a sequence of positive and identically distributed random variables (RVs) with common distribution function (DF) $F$ satisfying $\bar{F}(x)=1-F(x)>0$ for all $x>0$. In addition, the claim sizes are bivariate upper tail independent (see Section 2 for the definition). The interarrival times $\left\{Y_{n}, n \geq 1\right\}$, independent of $\left\{X_{n}, n \geq 1\right\}$, form another sequence of independent and identically distributed exponential RVs with parameter $\lambda>0$. Then, the arrival times of the successive claims, $\tau_{n}=\sum_{i=1}^{n} Y_{i}, n \geq 1$, constitute a homogeneous Poisson process

$$
N_{t}=\sum_{n=1}^{\infty} \mathbf{1}_{\left[\tau_{n} \leq t\right]}, \quad t \geq 0 .
$$

Here and in the sequel, $\mathbf{1}_{A}$ denotes the indicator function of an event $A$. Hence, the total claim

Received 4 May 2010; revision received 28 March 2012.

* Postal address: Center of Financial Engineering, Nanjing Audit University, Nanjing 211815, China.

Email address: wangdc@nau.edu.cn 
sizes up to time $t \geq 0$ can be expressed as

$$
S_{t}=\sum_{n=1}^{N_{t}} X_{n}
$$

with $S_{t}=0$ when $N_{t}=0$.

Suppose that the total premiums up to time $t \geq 0$ is $c t$, where $c$ is a positive constant, and the surplus process of the insurance business is perturbed by a diffusion. Then, the total surplus of the insurance business up to time $t \geq 0$, denoted by $R_{t}$, can be written as

$$
R_{t}=x+c t-S_{t}+\sigma B_{t},
$$

where $x>0$ is the initial capital, $\sigma>0$ is the constant volatility, and $B$ is a standard Brownian motion.

Suppose that the surplus of the insurance business can be invested into a portfolio of some risk-free and risky assets. The price process $\left\{Z_{t}, t \geq 0\right\}$ of the investment portfolio satisfies

$$
\mathrm{d} Z_{t}=Z_{t-} \mathrm{d} L_{t}, \quad t>0, Z_{0}=1,
$$

where $\left\{L_{t}, t \geq 0\right\}$ is a semimartingale. According to Theorem 37 of [24, Chapter II], the solution to the above stochastic differential equation (SDE) is

$$
Z_{t}=\exp \left\{L_{t}-\frac{1}{2}[L, L]_{t}\right\} \prod_{0<s \leq t}\left(1+\Delta L_{s}\right) \exp \left\{-\Delta L_{s}+\frac{1}{2}\left(\Delta L_{s}\right)^{2}\right\}=: \mathrm{e}^{\hat{L}_{t}},
$$

where $\Delta L_{s}=L_{s}-L_{s-}$ and $[L, L]$ is the quadratic variation process of $L$. Obviously, $\hat{L}$ is also a semimartingale. For more details of semimartingales and stochastic integrals, see [24].

Define the integrated risk process $\left\{U_{t}, t \geq 0\right\}$ as the result of the insurance business and the net gains of the investment, namely, the solution to the SDE

$$
\mathrm{d} U_{t}=U_{t-} \mathrm{d} L_{t}+\mathrm{d} R_{t}, \quad t>0, U_{0}=x .
$$

Provided that the quadratic covariation process $[L, R] \equiv 0$ almost surely, the solution is

$$
U_{t}=\mathrm{e}^{\hat{L}_{t}}\left(x+\int_{0}^{t} \mathrm{e}^{-\hat{L}_{s-}} \mathrm{d} R_{s}\right), \quad t>0, U_{0}=x .
$$

In this paper, instead of the semimartingale $\left\{\hat{L}_{t}, t \geq 0\right\}$, we adopt a more general adapted càdlàg process $\left\{\xi_{t}, t \geq 0\right\}$ with $\xi_{0}=0$, independent of $\left\{X_{n}, n \geq 1\right\}$ and $\left\{\tau_{n}, n \geq 1\right\}$, to model the log investment returns. Namely, we consider the more general risk model

$$
U_{t}=\mathrm{e}^{\xi_{t}}\left(x+\int_{0}^{t} \mathrm{e}^{-\xi_{s-}} \mathrm{d} R_{s}\right), \quad t>0, U_{0}=x .
$$

The ruin probability up to time $t \geq 0$ and the infinite-time ruin probability of model (1.1) are respectively defined as

$$
\Psi(x, t)=\mathbb{P}\left(\bigcup_{0 \leq s \leq t}\left(U_{s}<0\right) \mid U_{0}=x\right), \quad \Psi(x)=\mathbb{P}\left(\bigcup_{0 \leq s<\infty}\left(U_{s}<0\right) \mid U_{0}=x\right) .
$$


Many researchers have studied Poisson risk models with risky investments. For example, Frolova et al. [13], Paulsen and Gjessing [23], Kalashnikov and Norberg [17], and Gaier and Grandits [14] considered Poisson risk models with investment returns modeled by geometric Brownian motions. With the prices of risky assets modeled by general exponential Lévy processes with jumps, Emmer and Klüppelberg [12] investigated the optimization problem of investment portfolios. Paulsen [22] considered the asymptotic behavior for large initial capital of the ultimate ruin probability under a Poisson risk model when investment returns are a general Lévy process. His result showed that the ruin probability behaves like a Pareto function of the (large) initial capital. The Pareto exponent depends on the interaction between insurance claims and the investment return process. Cai and Yang [6] investigated the ruin probabilities of a perturbed compound Poisson risk model in which investment returns follow a linear process or a jump diffusion process. Using the theory of stochastic recurrence equations (see [15] and [19]), Klüppelberg and Kostadinova [18] studied the tail behavior of the ultimate integrated risk for a discounted net loss process. They showed that if the order of the finite moment of the common claim size distribution function is greater than $\kappa_{\theta}$ (a constant related to portfolio investments but not to insurance claims; see their paper for details), the extreme of investment risk will determine the tail behavior of the ultimate integrated risk for the process. They referred to this as the case of dangerous investment. If the claim size distribution function has a regularly varying tail with tail index $-\alpha$ and $\alpha<\kappa_{\theta}$, the extreme of insurance risk will determine the tail behavior of the ultimate integrated risk for the process. They referred to this as the case of dangerous claims. Heyde and Wang [16] further investigated the finite-time ruin probability of the Poisson risk model considered by Klüppelberg and Kostadinova [18] when the claim size distribution function is in a larger heavy-tailed distribution function class. Their result showed that in finite time, however, the extreme of insurance risk dominates that of investment risk, but, for the case of dangerous investment, the extreme of investment risk has a greater effect on the total risk, and as time passes, the extreme of investment risk finally dominates the extreme of insurance risk.

Recently, the asymptotic tail probabilities for many discrete-time risk models with dependence structures have attracted a lot of attention due to their practical importance. Among them, Mikosch and Samorodnitsky [20] used a two-sided linear process to model the step sizes of a random walk and investigated the tail asymptotics for the supremum of the partial sums of the random walk. Under a regular variation tail condition, they showed that the dependence among the step sizes has an obvious impact on the tail asymptotics. However, the studies of Wang and Tang [27], Tang [26], and Wang et al. [29] showed that the tail asymptotics for the partial sums of random walks are insensitive to the case of negatively dependent or negatively associated step sizes under different heavy tail conditions. It should be noted that the effect of investment returns was not considered in any of these papers.

The asymptotic tail probabilities for randomly weighted sums $\sum_{i=1}^{n} \Theta_{i} X_{i}$ and their maxima, where the step sizes $\left\{X_{i}, i \geq 1\right\}$ are independent of random weights $\left\{\Theta_{i}, i \geq 1\right\}$, have important applications in discrete-time risk models. Wang and Tang [28] and Wang et al. [30] studied them under the condition that $\left\{X_{i}, i \geq 1\right\}$ is a sequence of independent and identically distributed RVs. Zhang et al. [31] extended their results to the case in which the step sizes $\left\{X_{i}, i \geq 1\right\}$ are bivariate upper tail independent. When the common distribution function of $\left\{X_{i}, i \geq 1\right\}$ has an extended regular varying tail, they proved that $\mathbb{P}\left(\max _{1 \leq k} \leq n \sum_{i=1}^{k} \Theta_{i} X_{i}>x\right) \sim \mathbb{P}\left(\sum_{i=1}^{n} \Theta_{i} X_{i}>x\right) \sim \sum_{i=1}^{n} \mathbb{P}\left(\Theta_{i} X_{i}>x\right)$ and extended them to the case $n=\infty$. In all of these papers the results were applied to the discussion of discrete-time risk models with random investment returns. 
Chen and $\mathrm{Ng}$ [7] investigated the asymptotic behavior of the ultimate ruin probability for a renewal risk model with constant interest rate and pairwise negatively dependent claim sizes. For the case of extended regularly varying tails, they proved that $\Psi(x) \sim \int_{0}^{\infty} \bar{F}\left(x \mathrm{e}^{\delta s}\right) \mathrm{d} \lambda_{s}$, where $\delta$ is the constant interest rate.

It is worth pointing out that in all Poisson risk models considered by Paulsen [22], Paulsen and Gjessing [23], Cai and Yang [6], Klüppleberg and Kostadinova [18], and Heyde and Wang [16], investment return processes are assumed to have independent and stationary increments and claim processes are modeled by homogeneous Poisson processes with independent claim sizes. However, empirical evidence shows that there is often a long-range dependence in square or absolute returns, and returns and their volatilities are often negatively correlated. Such evidence indicates that investment return processes usually have nonindependent or nonstationary increments. Besides, because of the increasing complexity of insurance and reinsurance products, it is more realistic to investigate insurance risk models with dependent claim sizes. There are few papers in which ruin problems of continuous-time risk models with dependence structures are investigated. Collamore [9] discussed random recurrence equations and the infinite-time ruin probability with a Markov-dependent stochastic investment return process, which still has a strong constraint on dependence structures.

Until now, no paper has simultaneously addressed continuous-time risk models with general stochastic investment returns and dependent claim sizes. In this paper we use bivariate upper tail independence introduced by Zhang et al. [31] to characterize the dependence structure of claim sizes and adopt a general adapted càdlàg process to model stochastic investment returns. When the claim size distribution function is heavy tailed and the investment returns satisfy some mild conditions, we derive some asymptotic formulae for the finite- and infinite-time ruin probabilities of model (1.1). We thus show that the asymptotic ruin probabilities are dominated by the extreme of insurance risk but not by that of investment risk. As applications of the results, we discuss four special cases when the investment returns are determined by a fractional Brownian motion, an integrated Vasicek model, an integrated Cox-Ingersoll-Ross model, and the Heston model.

The rest of this paper consists of four sections. In Section 2 we introduce some notation and state the main results in this paper. In Section 3 we discuss some applications of the main results. Section 4 provides some important lemmas, and in Section 5 we prove the main results.

\section{Notation and main results}

Hereafter, all limit relationships are for $x \rightarrow \infty$ unless stated otherwise. For two positive functions $a(\cdot)$ and $b(\cdot)$ satisfying

$$
0 \leq l^{-}=\liminf _{x \rightarrow \infty} \frac{a(x)}{b(x)} \leq \limsup _{x \rightarrow \infty} \frac{a(x)}{b(x)}=l^{+} \leq \infty,
$$

we write $a(x) \lesssim b(x)$ if $l^{+} \leq 1 ; a(x) \gtrsim b(x)$ if $l^{-} \geq 1 ; a(x) \sim b(x)$ if $l^{+}=l^{-}=1$; and $a(x) \asymp b(x)$ if $0<l^{-} \leq l^{+}<\infty$.

We recall two classes of heavy-tailed distribution functions which are crucial for our purpose. We say that a distribution function $F$ has a regularly varying tail with index $-\alpha \leq 0$, denoted by $F \in \mathcal{R}_{-\alpha}$, if

$$
\lim _{x \rightarrow \infty} \frac{\bar{F}(x y)}{\bar{F}(x)}=y^{-\alpha} \quad \text { for any } y>0
$$


We say that a distribution function $F$ belongs to the class $\mathcal{C}$ (has a consistently varying tail) if

$$
\lim _{c \uparrow 1} \limsup _{x \rightarrow \infty} \frac{\bar{F}(c x)}{\bar{F}(x)}=1, \quad \text { or, equivalently, } \quad \lim _{c \downarrow 1} \liminf _{x \rightarrow \infty} \frac{\bar{F}(c x)}{\bar{F}(x)}=1 .
$$

The regular property in (2.1) of the tail probability $\bar{F}$ was first introduced and named the 'intermediate regular varying property' by Bingham et al. [2]. The class $\mathcal{C}$ has been used in many studies of applied probability, such as queueing and risk theories, and contains the class $\mathcal{R}_{-\alpha}$. In fact, Cai and Tang [5] showed that the class $\mathcal{C}$ is strictly larger than the class $\mathcal{R}_{-\alpha}$.

For notational convenience, we also say that an RV belongs to one of the two classes if its distribution function belongs to the class.

Now we recall two significant indices of a general RV. Let $X$ be an RV with a distribution function $F$ concentrated on $(-\infty, \infty)$. For any $y>0$, we set

$$
\bar{F}_{*}(y)=\liminf _{x \rightarrow \infty} \frac{\bar{F}(x y)}{\bar{F}(x)} \quad \text { and } \quad \bar{F}^{*}(y)=\limsup _{x \rightarrow \infty} \frac{\bar{F}(x y)}{\bar{F}(x)},
$$

and then define

$$
\begin{aligned}
& \mathbb{J}_{F}^{+}=\mathbb{J}^{+}(X)=\inf \left\{-\frac{\log \bar{F}_{*}(y)}{\log y}: y>1\right\}=-\lim _{y \rightarrow \infty} \frac{\log \bar{F}_{*}(y)}{\log y}, \\
& \mathbb{J}_{F}^{-}=\mathbb{J}^{-}(X)=\sup \left\{-\frac{\log \bar{F}^{*}(y)}{\log y}: y>1\right\}=-\lim _{y \rightarrow \infty} \frac{\log \bar{F}^{*}(y)}{\log y} .
\end{aligned}
$$

Here $\mathbb{J}_{F}^{+}$and $\mathbb{J}_{F}^{-}$are called the upper and lower Matuszewska indices of the nonnegative and nondecreasing function $f(x)=(\bar{F}(x))^{-1}, x \geq 0$ (see Chapter 2.1 of [2]). Specifically, if $F \in \mathcal{C}$ then $0 \leq \mathbb{J}_{F}^{+}<\infty$, and if $F \in \mathcal{R}_{-\alpha}$ with $\alpha \geq 0$ then $\mathbb{J}_{F}^{-}=\mathbb{J}_{F}^{+}=\alpha$.

Next, we give the definition of the bivariate upper tail independence of a random sequence, which was also considered by Zhang et al. [31]. We say that a random sequence $\left\{X_{n}, n \geq 1\right\}$ is bivariate upper tail independent or has no bivariate upper tail dependence if, for any $i, j \geq 1$, $i \neq j$,

$$
\lim _{x \rightarrow \infty} \frac{\mathbb{P}\left(X_{i}>x, X_{j}>x\right)}{\mathbb{P}\left(X_{k}>x\right)}=0, \quad k=i, j .
$$

We are now ready to state the main results in this paper.

Theorem 2.1. Consider the Poisson risk model introduced in Section 1 with $F \in \mathcal{C}$ and claim sizes satisfying (2.2). If there exists some constant $\kappa>\max \left\{\mathbb{J}_{F}^{+}, 2\right\}$ such that $\int_{0}^{t} \mathbb{E} \mathrm{e}^{-\kappa \xi_{s}} \mathrm{~d} s<\infty$ for some $t>0$ then

$$
\Psi(x, t) \sim \lambda \int_{0}^{t} \mathbb{P}\left(X_{1} \mathrm{e}^{-\xi_{s}}>x\right) \mathrm{d} s \quad \text { as } x \rightarrow \infty .
$$

Specifically, if $F \in \mathcal{R}_{-\alpha}$ for some $\alpha \geq 0$ then

$$
\Psi(x, t) \sim \lambda \bar{F}(x) \int_{0}^{t} \mathbb{E} \mathrm{e}^{-\alpha \xi_{s}} \mathrm{~d} s \quad \text { as } x \rightarrow \infty .
$$


Theorem 2.2. Consider the Poisson risk model introduced in Section 1. Suppose that $F \in \mathcal{C}$ with $\mathbb{J}_{F}^{-}>0$ and that the claim sizes satisfy (2.2). If there exist some constants $\kappa>\max \left\{\mathbb{J}_{F}^{+}, 2\right\}$ and $\eta>\kappa-1+2 \kappa / \mathbb{J}_{F}^{-}$such that $\int_{0}^{\infty} \max \left\{s^{\eta}, 1\right\} \mathbb{E} \mathrm{e}^{-\kappa \xi_{s}} \mathrm{~d} s<\infty$, then

$$
\Psi(x) \sim \lambda \int_{0}^{\infty} \mathbb{P}\left(X_{1} \mathrm{e}^{-\xi_{s}}>x\right) \mathrm{d} s \quad \text { as } x \rightarrow \infty .
$$

Specifically, if $F \in \mathcal{R}_{-\alpha}$ for some $\alpha>0$ then

$$
\Psi(x) \sim \lambda \bar{F}(x) \int_{0}^{\infty} \mathbb{E}^{-\alpha \xi_{s}} \mathrm{~d} s \quad \text { as } x \rightarrow \infty .
$$

Remark 2.1. Theorems 2.1 and 2.2 address only the cases in which the asymptotic ruin probability is dominated by the extreme of insurance risk but not by that of investment risk. This can be seen from relations (2.4) and (2.6), which show that the tail probability of the claim sizes determines the exact decay rate, while the investment uncertainty and the claim frequency contribute to only the coefficients of these asymptotic formulae. It is well known that if claim sizes do not have such heavy tails, the investment risk will dominate the ruin asymptotics.

Remark 2.2. If $\left\{X_{n}, n \geq 1\right\}$ is a sequence of independent and identically distributed RVs, assumption (2.2) will be satisfied automatically and the corresponding results of Klüppelberg and Kostadinova [18] and Heyde and Wang [16] can be derived from Theorems 2.1 and 2.2 with $\xi_{s}$ replaced by a Lévy process $L_{\theta}(s)$ specified in their papers.

Remark 2.3. Assumption (2.2) is closely related to the copula-based index of upper tail dependence. Let $\boldsymbol{X}=\left(X_{1}, X_{2}\right)$ be a two-dimensional random vector with continuous and strongly increasing marginal distribution functions $F_{X_{1}}$ and $F_{X_{2}}$. The index of the upper tail dependence for $\boldsymbol{X}$ is defined as

$$
\begin{aligned}
\lambda_{\mathrm{U}} & =\lim _{v \uparrow 1} \frac{\mathbb{P}\left(X_{1}>F_{X_{1}}^{-1}(v), X_{2}>F_{X_{2}}^{-1}(v)\right)}{\mathbb{P}\left(X_{k}>F_{X_{k}}^{-1}(v)\right)} \\
& =\lim _{v \uparrow 1} \frac{\mathbb{P}\left(X_{1}>F_{X_{1}}^{-1}(v), X_{2}>F_{X_{2}}^{-1}(v)\right)}{1-v}, \quad k=1,2,
\end{aligned}
$$

provided it exists. We say that $\boldsymbol{X}$ has upper tail dependence if $\lambda_{U} \in(0,1]$, and has no upper tail dependence if $\lambda_{\mathrm{U}}=0$. If the claim sizes $\left\{X_{n}, n \geq 1\right\}$ are identically distributed then Sklar's theorem (see, e.g. [21]) ensures that assumption (2.2) is equivalent to no upper tail dependence.

\section{Applications}

In this section we apply our results to some important investment return processes.

\subsection{Application to fractional Brownian motions}

Suppose that the investment return process $\left\{Z_{t}=\mathrm{e}^{\xi_{t}}, t \geq 0\right\}$ is modeled by

$$
\mathrm{d} Z_{t}=\mu Z_{t} \mathrm{~d} t+\delta Z_{t} \mathrm{~d} B_{H}(t), \quad t>0, Z_{0}=1
$$

where $\mu, \delta>0$ are two constants and $\left\{B_{H}(t), t \geq 0\right\}$ is a fractional Brownian motion with Hurst parameter $H \in(0,1)$. By definition, a process $\left\{B_{H}(t), t \geq 0\right\}$ is said to be a 
fractional Brownian motion if it is a continuous-time Gaussian process starting from 0 with mean $\mathbb{E} B_{H}(t)=0$ for all $t \geq 0$ and covariance

$$
\mathbb{E}\left[B_{H}(t) B_{H}(s)\right]=\frac{1}{2}\left(|t|^{2 H}+|s|^{2 H}-|t-s|^{2 H}\right), \quad t, s \geq 0 .
$$

The integral form of (3.1) is

$$
Z_{t}=1+\mu \int_{0}^{t} Z_{s} \mathrm{~d} s+\delta \int_{0}^{t} Z_{s} \mathrm{~d} B_{H}(s),
$$

where $\int_{0}^{t} Z_{s} \mathrm{~d} B_{H}(s)$ is a Wick-Itô-Skorokhod integral. For more details of fractional Brownian motions and Wick-Itô-Skorokhod integrals, see [1].

The following theorem is a consequence of Theorems 2.1 and 2.2.

Theorem 3.1. Consider the Poisson risk model introduced in Section 1. Suppose that the investment return process $\left\{Z_{t}=\mathrm{e}^{\xi_{t}}, t \geq 0\right\}$ is modeled by (3.1) or (3.2) with Hurst parameter $H \in(0,1)$, and that the claim sizes $\left\{X_{n}, n \geq 1\right\}$ are bivariate upper tail independent, i.e. they satisfy relation (2.2). If the claim-size distribution function $F \in \mathcal{R}_{-\alpha}$ for some $\alpha>0$ then, for arbitrarily fixed $t>0$,

$$
\Psi(x, t) \sim \lambda \bar{F}(x) \int_{0}^{t} \exp \left\{-\alpha \mu s+\frac{\delta^{2}\left(\alpha+\alpha^{2}\right)}{2} s^{2 H}\right\} \mathrm{d} s \quad \text { as } x \rightarrow \infty .
$$

Furthermore, for the case $H \in\left(0, \frac{1}{2}\right)$, we have

$$
\Psi(x) \sim \lambda \bar{F}(x) \int_{0}^{\infty} \exp \left\{-\alpha \mu s+\frac{\delta^{2}\left(\alpha+\alpha^{2}\right)}{2} s^{2 H}\right\} \mathrm{d} s \quad \text { as } x \rightarrow \infty ;
$$

for the case $H=\frac{1}{2}$, if $\delta^{2}(\max \{\alpha, 2\}+1)<2 \mu$ then relation (3.4) still holds.

Proof. By Itô's formula for Wick-Itô-Skorokhod integrals (see, e.g. Theorem 4.2.6 of [1]), we can verify that the solution to (3.1) or (3.2) is

$$
\mathrm{e}^{\xi_{t}}=Z_{t}=\exp \left\{\mu t+\delta B_{H}(t)-\frac{1}{2} \delta^{2} t^{2 H}\right\}
$$

Note that $B_{H}(t)$ is a Gaussian RV with mean $\mathbb{E} B_{H}(t)=0$ and variance $\operatorname{var}\left(B_{H}(t)\right)=t^{2 H}$. Then, for any $\kappa>0$, we have

$$
\begin{aligned}
\mathbb{E} \mathrm{e}^{-\kappa \xi_{t}} & =\mathbb{E} \exp \left\{-\kappa \mu t-\kappa \delta B_{H}(t)+\frac{\kappa}{2} \delta^{2} t^{2 H}\right\} \\
& =\exp \left\{-\kappa \mu t+\frac{\delta^{2}\left(\kappa+\kappa^{2}\right)}{2} t^{2 H}\right\} .
\end{aligned}
$$

It follows that $\int_{0}^{t} \mathbb{E} \mathrm{e}^{-\kappa \xi_{s}} \mathrm{~d} s<\infty$ for any $\kappa>0$. By Theorem 2.1 and (3.6), relation (3.3) holds.

Next, we prove relation (3.4). For the case $H \in\left(0, \frac{1}{2}\right)$, from (3.6) we obtain $\int_{0}^{\infty} \max \left\{s^{\eta}, 1\right\}$ $\mathbb{E} \mathrm{e}^{-\kappa \xi_{s}} \mathrm{~d} s<\infty$ for any $\kappa>0$ and any $\eta>0$. According to Theorem 2.2 and (3.6), we obtain (3.4). For the case $H=\frac{1}{2}$, since $\delta^{2}(\max \{\alpha, 2\}+1)<2 \mu$, we can take some $\kappa>\max \{\alpha, 2\}$ such that

$$
-\kappa \mu+\frac{1}{2} \delta^{2}\left(\kappa+\kappa^{2}\right)<0 .
$$

For the fixed $\kappa$ and any $\eta>0$, from (3.6) we obtain $\int_{0}^{\infty} \max \left\{s^{\eta}, 1\right\} \mathbb{E} \mathrm{e}^{-\kappa \xi_{s}} \mathrm{~d} s<\infty$. Thus, by Theorem 2.2 and (3.6), we obtain (3.4). 
Remark 3.1. If $H>\frac{1}{2}$ then $\left\{B_{H}(t), t \geq 0\right\}$ has a long-range dependence in the sense that

$$
\sum_{n=1}^{\infty} \varrho(n)=\infty
$$

with $\varrho(n)=\operatorname{cov}\left\{B_{H}(1),\left[B_{H}(n+1)-B_{H}(n)\right]\right\}$. Define $\varrho \xi(n)=\operatorname{cov}\left\{\xi_{1},\left(\xi_{n+1}-\xi_{n}\right)\right\}$, where the $\xi_{t}, t \geq 0$, are defined in (3.5). From $\mathbb{E} B_{H}(t)=0$ we obtain

$$
\varrho \xi(n)=\delta^{2} \mathbb{E}\left(B_{H}(1)\left[B_{H}(n+1)-B_{H}(n)\right]\right)=\delta^{2} \varrho(n) .
$$

Hence, $\sum_{n=1}^{\infty} \varrho \xi(n)=\delta^{2} \sum_{n=1}^{\infty} \varrho(n)=\infty$, namely, the log-investment return process $\left\{\xi_{t}, t \geq\right.$ $0\}$ has a long-range dependence. This is different from the risk model considered by Klüppelberg and Kostadinova [18] and Heyde and Wang [16] in which the log returns of portfolio investment are Lévy processes and, hence, have independent increments.

\subsection{Application to integrated stochastic short rate models}

Suppose that the log-investment return process $\left\{\xi_{t}, t \geq 0\right\}$ satisfies

$$
\xi_{t}=\int_{0}^{t} r_{s} \mathrm{~d} s, \quad t>0, \xi_{0}=0,
$$

where $\left\{r_{t}, t \geq 0\right\}$ is a stochastic short-rate process satisfying

$$
\mathrm{d} r_{t}=\gamma\left(\iota-r_{t}\right) \mathrm{d} t+\delta r_{t}^{\pi} \mathrm{d} W_{t} .
$$

Here $\gamma, \iota$, and $\delta$ are three positive constants, $\pi=0$ or $\frac{1}{2}$, and $\left\{W_{t}, t \geq 0\right\}$ is a standard Wiener process. In mathematical finance, model (3.8) with $\pi=0$ is called the Vasicek model and model (3.8) with $\pi=\frac{1}{2}$ is called the Cox-Ingersoll-Ross model.

We first consider the case in which $\left\{\xi_{t}, t \geq 0\right\}$ is modeled by (3.7)-(3.8) with $\pi=0$, namely, the log-investment return process is an integrated Vasicek process. In this case, the following theorem holds.

Theorem 3.2. Consider the Poisson risk model introduced in Section 1. Suppose that the loginvestment return process $\left\{\xi_{t}, t \geq 0\right\}$ is modeled by (3.7)-(3.8) with $\pi=0$ and that the claim sizes $\left\{X_{n}, n \geq 1\right\}$ are bivariate upper tail independent, i.e. they satisfy relation (2.2). If the claim size distribution function $F \in \mathcal{R}_{-\alpha}$ for some $\alpha>0$ then, for arbitrarily fixed $t>0$,

$$
\Psi(x, t) \sim \lambda \bar{F}(x) \int_{0}^{t} \exp \left\{A_{1}(\alpha)+A_{2}(\alpha) s+A_{3}(\alpha) \mathrm{e}^{-\gamma s}+A_{4}(\alpha) \mathrm{e}^{-2 \gamma s}\right\} \mathrm{d} s \quad \text { as } x \rightarrow \infty,
$$

where

$$
\begin{array}{ll}
A_{1}(\alpha)=\frac{\iota-r_{0}}{\gamma} \alpha-\frac{3 \delta^{2}}{4 \gamma^{3}} \alpha^{2}, & A_{2}(\alpha)=-\iota \alpha+\frac{\delta^{2}}{2 \gamma^{2}} \alpha^{2}, \\
A_{3}(\alpha)=\frac{r_{0}-\iota}{\gamma} \alpha+\frac{\delta^{2}}{\gamma^{3}} \alpha^{2}, & A_{4}(\alpha)=-\frac{\delta^{2}}{4 \gamma^{3}} \alpha^{2} .
\end{array}
$$

Furthermore, if $\max \{\alpha, 2\}<2 \gamma^{2} \iota / \delta^{2}$ then

$$
\Psi(x) \sim \lambda \bar{F}(x) \int_{0}^{\infty} \exp \left\{A_{1}(\alpha)+A_{2}(\alpha) s+A_{3}(\alpha) \mathrm{e}^{-\gamma s}+A_{4}(\alpha) \mathrm{e}^{-2 \gamma s}\right\} \mathrm{d} s \text { as } x \rightarrow \infty .
$$


Proof. We will apply Theorems 2.1 and 2.2 to prove this theorem. To verify the conditions of Theorems 2.1 and 2.2, we need the explicit expression of $\mathbb{E} \mathrm{e}^{-\kappa \xi_{t}}$. Instead of computing $\mathbb{E} \mathrm{e}^{-\kappa \xi_{t}}$ directly, we compute the moment generating function of $\left(r_{t}, \xi_{t}\right)$. For any pair of real numbers $(a, b)$, define

$$
M_{1}(a, b, t)=\mathbb{E} \mathrm{e}^{a r_{t}+b \xi_{t}}=\mathbb{E} \exp \left\{a r_{t}+b \int_{0}^{t} r_{s} \mathrm{~d} s\right\},
$$

where the $r_{t}, t \geq 0$, are defined in (3.8) with $\pi=0$. According to Example 4.4.10 of [25], $r_{t}$ is a Gaussian RV. Then, $\int_{0}^{t} r_{s} \mathrm{~d} s=\left(\gamma \iota t+r_{0}-r_{t}+\delta W_{t}\right) / \gamma$ is also a Gaussian random variable. Thus, $M_{1}(a, b, t)$ is finite for any real vector $(a, b)$. Applying Itô's formula to $\exp \left\{a r_{t}+b \int_{0}^{t} r_{s} \mathrm{~d} s\right\}$ and then taking expectations, we obtain

$$
M_{1}(a, b, t)=\mathrm{e}^{a r_{0}}+\left(a \gamma \iota+\frac{a^{2} \delta^{2}}{2}\right) \int_{0}^{t} M_{1}(a, b, s) \mathrm{d} s+(b-a \gamma) \int_{0}^{t} \frac{\partial M_{1}(a, b, s)}{\partial a} \mathrm{~d} s,
$$

where we have used the following property of the moment generating function:

$$
\frac{\partial M_{1}(a, b, s)}{\partial a}=\mathbb{E}\left(r_{s} \exp \left\{a r_{s}+b \int_{0}^{s} r_{v} \mathrm{~d} v\right\}\right) .
$$

Differentiating the above integral equation with respect to $t$, we obtain

$$
\begin{gathered}
\frac{\partial M_{1}(a, b, t)}{\partial t}+(a \gamma-b) \frac{\partial M_{1}(a, b, t)}{\partial a}=\left(a \gamma \iota+\frac{a^{2} \delta^{2}}{2}\right) M_{1}(a, b, t), \\
M_{1}(a, b, 0)=\mathrm{e}^{a r_{0}} .
\end{gathered}
$$

Now we apply the method of characteristics to solve (3.11). The characteristic equations of (3.11) are

$$
\frac{\mathrm{d}}{\mathrm{d} s} t(c, s)=1, \quad \frac{\mathrm{d}}{\mathrm{d} s} a(c, s)=a \gamma-b, \quad \frac{\mathrm{d}}{\mathrm{d} s} z(c, s)=a \gamma \iota z+\frac{a^{2} \delta^{2}}{2} z,
$$

with initial conditions

$$
t(c, 0)=0, \quad a(c, 0)=c, \quad z(c, 0)=\mathrm{e}^{c r_{0}} .
$$

The solution to (3.12)-(3.13) is

$$
\begin{gathered}
t(c, s)=s, \quad a(c, s)=\frac{b}{\gamma}+\left(c-\frac{b}{\gamma}\right) \mathrm{e}^{\gamma s} \\
z(c, s)=\exp \left\{c r_{0}+\gamma \iota \int_{0}^{s} a(c, y) \mathrm{d} y+\frac{\delta^{2}}{2} \int_{0}^{s} a^{2}(c, y) \mathrm{d} y\right\} .
\end{gathered}
$$

From (3.14), we can solve for $c$ and $s$ in terms of $a$ and $t$ :

$$
s=t, \quad c=\frac{b}{\gamma}+\left(a-\frac{b}{\gamma}\right) \mathrm{e}^{-\gamma t} .
$$

Thus, by (3.14)-(3.15), we obtain

$$
\begin{aligned}
M_{1}(a, b, t) & =z(c(a, t), s(a, t)) \\
& =\exp \left\{\hat{A}_{1}(a, b)+\hat{A}_{2}(a, b) t+\hat{A}_{3}(a, b) \mathrm{e}^{-\gamma t}+\hat{A}_{4}(a, b) \mathrm{e}^{-2 \gamma t}\right\},
\end{aligned}
$$


where

$$
\begin{gathered}
\hat{A}_{1}(a, b)=\frac{b r_{0}}{\gamma}+\left(\iota+\frac{b \delta^{2}}{\gamma^{2}}\right)\left(a-\frac{b}{\gamma}\right)+\frac{\delta^{2}}{4 \gamma}\left(a-\frac{b}{\gamma}\right)^{2}, \quad \hat{A}_{2}(a, b)=b \iota+\frac{\delta^{2} b^{2}}{2 \gamma^{2}}, \\
\hat{A}_{3}(a, b)=\left(r_{0}-\imath-\frac{b \delta^{2}}{\gamma^{2}}\right)\left(a-\frac{b}{\gamma}\right), \quad \hat{A}_{4}(a, b)=-\frac{\delta^{2}}{4 \gamma}\left(a-\frac{b}{\gamma}\right)^{2} .
\end{gathered}
$$

Now we are ready to compute $\mathbb{E} \mathrm{e}^{-\kappa \xi_{t}}$. By taking $a=0$ and $b=-\kappa$ in (3.16)-(3.18), we obtain

$$
\mathbb{E} \mathrm{e}^{-\kappa \xi_{t}}=M_{1}(0,-\kappa, t)=\exp \left\{A_{1}(\kappa)+A_{2}(\kappa) t+A_{3}(\kappa) \mathrm{e}^{-\gamma t}+A_{4}(\kappa) \mathrm{e}^{-2 \gamma t}\right\}
$$

where

$$
\begin{array}{ll}
A_{1}(\kappa)=\frac{\iota-r_{0}}{\gamma} \kappa-\frac{3 \delta^{2}}{4 \gamma^{3}} \kappa^{2}, & A_{2}(\kappa)=-\iota \kappa+\frac{\delta^{2}}{2 \gamma^{2}} \kappa^{2}, \\
A_{3}(\kappa)=\frac{r_{0}-\iota}{\gamma} \kappa+\frac{\delta^{2}}{\gamma^{3}} \kappa^{2}, & A_{4}(\kappa)=-\frac{\delta^{2}}{4 \gamma^{3}} \kappa^{2} .
\end{array}
$$

It follows that $\int_{0}^{t} \mathbb{E} \mathrm{e}^{-\kappa \xi_{s}} \mathrm{~d} s<\infty$ for any $\kappa>0$. By Theorem 2.1 and (3.19)-(3.21), we obtain (3.9).

Next, we prove relation (3.10). According to (3.19)-(3.21), we obtain

$$
\mathbb{E} \mathrm{e}^{-\kappa \xi_{t}} \sim \exp \left\{\frac{\iota-r_{0}}{\gamma} \kappa-\frac{3 \delta^{2}}{4 \gamma^{3}} \kappa^{2}+\left(-\kappa \iota+\frac{\delta^{2} \kappa^{2}}{2 \gamma^{2}}\right) t\right\} \quad \text { as } t \rightarrow \infty .
$$

Since $\max \{\alpha, 2\}<2 \gamma^{2} \iota / \delta^{2}$, we can take some $\kappa>0$ such that $\max \{\alpha, 2\}<\kappa<2 \gamma^{2} \iota / \delta^{2}$. Clearly, $-\kappa \iota+\delta^{2} \kappa^{2} /\left(2 \gamma^{2}\right)<0$. Thus, from the above asymptotic relation, we see that $\int_{0}^{\infty} \max \left\{s^{\eta}, 1\right\} \mathbb{E} \mathrm{e}^{-\kappa \xi_{s}} \mathrm{~d} s<\infty$ for the fixed $\kappa$ and any $\eta>0$. By Theorem 2.2 and (3.19)(3.21), we obtain (3.10).

Let us now consider the case in which $\left\{\xi_{t}, t \geq 0\right\}$ is modeled by (3.7)-(3.8) with $\pi=\frac{1}{2}$, namely, the log-investment return process is an integrated Cox-Ingersoll-Ross process. In this case, we have the following result.

Theorem 3.3. Consider the Poisson risk model introduced in Section 1. Suppose that the log-return process $\left\{\xi_{t}, t \geq 0\right\}$ is modeled by (3.7)-(3.8) with $\pi=\frac{1}{2}$ and that the claim sizes $\left\{X_{n}, n \geq 1\right\}$ are bivariate upper tail independent, i.e. they satisfy relation (2.2). If the claim size distribution function $F \in \mathcal{R}_{-\alpha}$ for some $\alpha>0$ then, for arbitrarily fixed $t>0$,

$$
\Psi(x, t) \sim \lambda \bar{F}(x) \int_{0}^{t} B_{1}(\alpha, s) \mathrm{e}^{B_{2}(\alpha, s) r_{0}} \mathrm{~d} s \quad \text { as } x \rightarrow \infty,
$$

where

$$
\begin{aligned}
\Omega(\alpha) & =\sqrt{\gamma^{2}+2 \delta^{2} \alpha} \\
B_{1}(\alpha, s) & =\left(\frac{\mathrm{e}^{\gamma s / 2}}{\cosh (\Omega(\alpha) s / 2)+\gamma \sinh (\Omega(\alpha) s / 2) / \Omega(\alpha)}\right)^{2 \gamma \iota / \delta^{2}} \\
B_{2}(\alpha, s) & =-\frac{2 \alpha}{\gamma+\Omega(\alpha) \operatorname{coth}(\Omega(\alpha) s / 2)}
\end{aligned}
$$


Furthermore,

$$
\Psi(x) \sim \lambda \bar{F}(x) \int_{0}^{\infty} B_{1}(\alpha, s) \mathrm{e}^{B_{2}(\alpha, s) r_{0}} \mathrm{~d} s \quad \text { as } x \rightarrow \infty .
$$

Proof. We will apply Theorems 2.1 and 2.2 to prove this theorem. To verify the conditions of Theorems 2.1 and 2.2, we need the explicit expression of $\mathbb{E} \mathrm{e}^{-\kappa \xi_{t}}$. Instead of computing $\mathbb{E} \mathrm{e}^{-\kappa \xi_{t}}$ directly, we compute the moment generating function of $\left(r_{t}, \xi_{t}\right)$. For any pair of real numbers $(a, b)$, define

$$
M_{2}(a, b, t)=\mathbb{E} \mathrm{e}^{a r_{t}+b \xi_{t}}=\mathbb{E} \exp \left\{a r_{t}+b \int_{0}^{t} r_{s} \mathrm{~d} s\right\}
$$

where the $r_{t}, t \geq 0$, are defined in (3.8) with $\pi=\frac{1}{2}$. Since both $r_{t}$ and $\int_{0}^{t} r_{s} \mathrm{~d} s$ have moment generating functions (see [11]), $M_{2}(a, b, t)$ is finite for $(a, b)$ in a neighborhood of $(0,0)$. Applying Itô's formula to $\exp \left\{a r_{t}+b \int_{0}^{t} r_{s} \mathrm{~d} s\right\}$ and then taking expectations, we obtain

$$
M_{2}(a, b, t)=\mathrm{e}^{a r_{0}}+a \gamma \iota \int_{0}^{t} M_{2}(a, b, s) \mathrm{d} s+\left(b+\frac{a^{2} \delta^{2}}{2}-a \gamma\right) \int_{0}^{t} \frac{\partial M_{2}(a, b, s)}{\partial a} \mathrm{~d} s,
$$

where we have used the following property of the moment generating function:

$$
\frac{\partial M_{2}(a, b, s)}{\partial a}=\mathbb{E}\left(r_{s} \exp \left\{a r_{s}+b \int_{0}^{s} r_{v} \mathrm{~d} v\right\}\right) .
$$

Differentiating the above integral equation with respect to $t$, we obtain

$$
\begin{gathered}
\frac{\partial M_{2}(a, b, t)}{\partial t}+\left(-b-\frac{a^{2} \delta^{2}}{2}+a \gamma\right) \frac{\partial M_{2}(a, b, t)}{\partial a}=a \gamma \iota M_{2}(a, b, t), \\
M_{2}(a, b, 0)=\mathrm{e}^{a r_{0}} .
\end{gathered}
$$

Now we apply the method of characteristics to solve (3.24). The characteristic equations of (3.24) are

$$
\frac{\mathrm{d}}{\mathrm{d} s} t(c, s)=1, \quad \frac{\mathrm{d}}{\mathrm{d} s} a(c, s)=-b-\frac{a^{2} \delta^{2}}{2}+a \gamma, \quad \frac{\mathrm{d}}{\mathrm{d} s} z(c, s)=a \gamma \iota z,
$$

with initial conditions

$$
t(c, 0)=0, \quad a(c, 0)=c, \quad z(c, 0)=\mathrm{e}^{c r_{0}} .
$$

Provided that $\gamma^{2}>2 \delta^{2} b$, the solution to (3.25)-(3.26) is

$$
\begin{aligned}
t(c, s)=s, \quad a(c, s) & =\frac{\gamma}{\delta^{2}}+\frac{\hat{\Omega}(b)}{\delta^{2}} \tanh \left(\frac{\hat{\Omega}(b) s}{2}-\operatorname{arctanh} \frac{\gamma-c \delta^{2}}{\hat{\Omega}(b)}\right), \\
z(c, s) & =\exp \left\{c r_{0}+\gamma \iota \int_{0}^{s} a(c, y) \mathrm{d} y\right\}
\end{aligned}
$$

where

$$
\hat{\Omega}(b)=\sqrt{\gamma^{2}-2 \delta^{2} b} .
$$


By (3.27), we can solve for $c$ and $s$ in terms of $a$ and $t$ :

$$
s=t, \quad c=\frac{\gamma}{\delta^{2}}-\frac{\hat{\Omega}(b)}{\delta^{2}} \tanh \left(\frac{\hat{\Omega}(b) t}{2}+\operatorname{arctanh} \frac{\gamma-a \delta^{2}}{\hat{\Omega}(b)}\right) .
$$

From (3.27)-(3.29), it follows that if $\gamma^{2}>2 \delta^{2} b$ then

$$
\begin{aligned}
M_{2}(a, b, t) & =z(c(a, t), s(a, t)) \\
& =\exp \left\{\hat{c}(a, b) r_{0}+\frac{(\gamma-\hat{\Omega}(b)) \gamma \iota t}{\delta^{2}}-\frac{2 \gamma \iota}{\delta^{2}} \ln \left(\frac{\zeta(a, b)-\mathrm{e}^{-\hat{\Omega}(b) t}}{\zeta(a, b)-1}\right)\right\},
\end{aligned}
$$

where $\hat{c}(a, b)$ has an equivalent expression to that of $c$ in (3.29), namely,

$$
\begin{gathered}
\hat{c}(a, b)=\frac{\gamma-\hat{\Omega}(b)}{\delta^{2}}-\frac{2 \hat{\Omega}(b)}{\delta^{2}} \frac{1}{\zeta(a, b) \mathrm{e}^{\hat{\Omega}(b) t}-1} \\
\text { and } \zeta(a, b)=1-\frac{2 \hat{\Omega}(b)}{\delta^{2} a+(\hat{\Omega}(b)-\gamma)} .
\end{gathered}
$$

Now we are ready to compute $\mathbb{E} \mathrm{e}^{-\kappa \xi_{t}}$. By taking $a=0$ and $b=-\kappa$ in (3.30)-(3.31), we obtain

$$
\mathbb{E} \mathrm{e}^{-\kappa \xi_{t}}=M_{2}(0,-\kappa, t)=B_{1}(\kappa, t) \mathrm{e}^{B_{2}(\kappa, t) r_{0}},
$$

where

$$
\begin{aligned}
\Omega(\kappa) & =\sqrt{\gamma^{2}+2 \delta^{2} \kappa}, \\
B_{1}(\kappa, t) & =\left(\frac{\mathrm{e}^{\gamma t / 2}}{\cosh (\Omega(\kappa) t / 2)+\gamma \sinh (\Omega(\kappa) t / 2) / \Omega(\kappa)}\right)^{2 \gamma \iota / \delta^{2}}, \\
B_{2}(\kappa, t) & =-\frac{2 \kappa}{\gamma+\Omega(\kappa) \operatorname{coth}(\Omega(\kappa) t / 2)} .
\end{aligned}
$$

It follows that $\int_{0}^{t} \mathbb{E} \mathrm{e}^{-\kappa \xi_{s}} \mathrm{~d} s<\infty$ for any $\kappa>0$. By Theorem 2.1 and (3.32)-(3.35), we obtain (3.22).

Next, we prove relation (3.23). By (3.32)-(3.35), we obtain

$$
\mathbb{E} \mathrm{e}^{-\kappa \xi_{t}} \sim C_{0} \exp \left\{\frac{\gamma \iota}{\delta^{2}}(\gamma-\Omega(\kappa)) t\right\}=C_{0} \exp \left\{\frac{\gamma \iota}{\delta^{2}}\left(\gamma-\sqrt{\gamma^{2}+2 \delta^{2} \kappa}\right) t\right\} \quad \text { as } t \rightarrow \infty,
$$

where $C_{0}$ is a positive constant. It follows that $\int_{0}^{\infty} \max \left\{s^{\eta}, 1\right\} \mathbb{E} \mathrm{e}^{-\kappa \xi_{s}} \mathrm{~d} s<\infty$ for any $\kappa>0$ and any $\eta>0$. By Theorem 2.2 and (3.32)-(3.35), we obtain (3.23).

\subsection{Application to the Heston model}

Suppose that the investment return process $\left\{Z_{t}=\mathrm{e}^{-\xi_{t}}, t \geq 0\right\}$ is described by the Heston model, namely,

$$
\mathrm{d} Z_{t}=\mu Z_{t} \mathrm{~d} t+\sqrt{v_{t}} Z_{t} \mathrm{~d} W_{t}^{(1)}
$$

where $\mu \geq 0$ is the risk-free rate, $\left\{W_{t}^{(1)}, t \geq 0\right\}$ is a standard Wiener process, and $\left\{v_{t}, t \geq 0\right\}$ is the variance process satisfying

$$
\mathrm{d} v_{t}=\gamma\left(\iota-v_{t}\right) \mathrm{d} t+\delta \sqrt{v_{t}} \mathrm{~d} W_{t}^{(2)}, \quad t>0
$$


with a constant starting point $\nu_{0}>0$. Here $\iota>0$ is the long-term mean, $\gamma>0$ is the mean reverting speed, $\delta>0$ is the volatility of the variance process itself, and $\left\{W_{t}^{(2)}, t \geq 0\right\}$ is another standard Wiener process correlated to the Wiener process $\left\{W_{t}^{(1)}, t \geq 0\right\}$ in (3.36) by

$$
\mathrm{d} W_{t}^{(1)}=\rho \mathrm{d} W_{t}^{(2)}+\sqrt{1-\rho^{2}} \mathrm{~d} W_{t}^{(3)},
$$

where $\left\{W_{t}^{(3)}, t \geq 0\right\}$ is a standard Wiener process independent of $\left\{W_{t}^{(2)}, t \geq 0\right\}$ and $\rho \in[-1,1]$ is the correlation coefficient. A negative correlation $(\rho<0)$ between $\left\{W_{t}^{(1)}, t \geq 0\right\}$ and $\left\{W_{t}^{(2)}, t \geq 0\right\}$ is known as the leverage effect. In addition, we assume that the parameters in (3.37) satisfy the stability condition

$$
\frac{2 \gamma \iota}{\delta^{2}}>1
$$

This condition ensures that the variance process $\left\{v_{t}, t \geq 0\right\}$ remains positive, starting from a positive variance $v_{0}$; see [4].

According to Theorems 2.1 and 2.2, the following theorem holds.

Theorem 3.4. Consider the Poisson risk model introduced in Section 1. Suppose that the investment return process $\left\{Z_{t}=\mathrm{e}^{\xi_{t}}, t \geq 0\right\}$ is modeled by (3.36)-(3.39) with $\rho \in[-1,0]$ and $\nu_{0}$ being a positive constant, and that the claim sizes $\left\{X_{n}, n \geq 1\right\}$ are bivariate upper tail independent, i.e. they satisfy relation (2.2). If the claim size distribution function $F \in \mathcal{R}_{-\alpha}$ for some $\alpha>0$ and the constant $\alpha^{\prime}=\max \{\alpha, 2\}$ satisfies

$$
\gamma+\alpha^{\prime} \rho \delta>\delta \sqrt{\alpha^{\prime}\left(\alpha^{\prime}+1\right)}
$$

then, for arbitrarily fixed $t>0$,

$$
\Psi(x, t) \sim \lambda \bar{F}(x) \int_{0}^{t} C_{1}(\alpha, s) \mathrm{e}^{-\alpha \mu s+C_{2}(\alpha, s) v_{0}} \mathrm{~d} s \quad \text { as } x \rightarrow \infty,
$$

where

$$
\begin{aligned}
\Delta(\alpha) & =\sqrt{(\gamma+\alpha \rho \delta)^{2}-\delta^{2}\left(\alpha^{2}+\alpha\right)} \\
C_{1}(\alpha, s) & =\left(\frac{\mathrm{e}^{(\gamma+\alpha \rho \delta) s / 2}}{\cosh (\Delta(\alpha) s / 2)+(\gamma+\alpha \rho \delta) \sinh (\Delta(\alpha) s / 2) / \Delta(\alpha)}\right)^{2 \gamma \iota / \delta^{2}}, \\
C_{2}(\alpha, s) & =\frac{\alpha^{2}+\alpha}{(\gamma+\alpha \rho \delta)+\Delta(\alpha) \operatorname{coth}(\Delta(\alpha) s / 2)} .
\end{aligned}
$$

Furthermore, if the constant $\alpha^{\prime}=\max \{\alpha, 2\}$ also satisfies

$$
\gamma \iota\left(\left(\gamma+\rho \alpha^{\prime} \delta\right)-\sqrt{\left(\gamma+\rho \alpha^{\prime} \delta\right)^{2}-\delta^{2} \alpha^{\prime}\left(\alpha^{\prime}+1\right)}\right)<\alpha^{\prime} \delta^{2} \mu,
$$

then

$$
\Psi(x) \sim \lambda \bar{F}(x) \int_{0}^{\infty} C_{1}(\alpha, s) \mathrm{e}^{-\alpha \mu s+C_{2}(\alpha, s) \nu_{0}} \mathrm{~d} s \quad \text { as } x \rightarrow \infty .
$$

Proof. We will apply Theorems 2.1 and 2.2 to prove this theorem. To verify the conditions of Theorems 2.1 and 2.2, we need the explicit expression of $\mathbb{E} \mathrm{e}^{-\kappa \xi_{t}}=\mathbb{E} Z_{t}^{-\kappa}$. By Itô's formula, we can verify that the solution to (3.36) is

$$
\begin{aligned}
Z_{t} & =\exp \left\{\mu t+\int_{0}^{t} \sqrt{\nu_{s}} \mathrm{~d} W_{s}^{(1)}-\frac{1}{2} \int_{0}^{t} v_{s} \mathrm{~d} s\right\} \\
& =\exp \left\{\mu t-\frac{1}{2} \int_{0}^{t} v_{s} \mathrm{~d} s+\rho \int_{0}^{t} \sqrt{\nu_{s}} \mathrm{~d} W_{s}^{(2)}+\sqrt{1-\rho^{2}} \int_{0}^{t} \sqrt{v_{s}} \mathrm{~d} W_{s}^{(3)}\right\} .
\end{aligned}
$$


From (3.40), we can take some $\kappa>\alpha^{\prime}=\max \{\alpha, 2\}$ such that

$$
\gamma+\kappa \rho \delta>\delta \sqrt{\kappa(\kappa+1)}
$$

For the fixed $\kappa$, by (3.47), we can derive

$$
\begin{aligned}
\mathbb{E} Z_{t}^{-\kappa}= & \mathbb{E}\left(\mathbb{E}\left(Z_{t}^{-\kappa} \mid W_{s}^{(2)}, 0 \leq s \leq t\right)\right) \\
= & \mathrm{e}^{-\kappa \mu t} \mathbb{E}\left(\exp \left\{\frac{\kappa}{2} \int_{0}^{t} v_{s} \mathrm{~d} s-\kappa \rho \int_{0}^{t} \sqrt{v_{s}} \mathrm{~d} W_{s}^{(2)}\right\}\right. \\
& \left.\quad \times \mathbb{E}\left(\exp \left\{-\kappa \sqrt{1-\rho^{2}} \int_{0}^{t} \sqrt{v_{s}} \mathrm{~d} W_{s}^{(3)}\right\} \mid W_{s}^{(2)}, 0 \leq s \leq t\right)\right) \\
= & : \mathrm{e}^{-\kappa \mu t} \mathbb{E}\left(\exp \left\{\frac{\kappa}{2} \int_{0}^{t} v_{s} \mathrm{~d} s-\kappa \rho \int_{0}^{t} \sqrt{v_{s}} \mathrm{~d} W_{s}^{(2)}\right\} K_{1}(t)\right) .
\end{aligned}
$$

Now we apply Girsanov's theorem to deal with $K_{1}(t)$. Note that $\left\{v_{t}, t \geq 0\right\}$ in (3.37) is actually a Cox-Ingersoll-Ross process. Then, the moment generating function of $\left(v_{t}, \int_{0}^{t} v_{s} \mathrm{~d} s\right)$ admits a similar expression in a certain neighborhood of $(0,0)$ to that of $\left(r_{t}, \int_{0}^{t} r_{s} \mathrm{~d} s\right)$ in (3.30)-(3.31) but with a different starting point $\nu_{0}$. By (3.48) and $\rho \in[-1,0]$, we have $\gamma^{2}>2 \delta^{2} b_{1}$ when $b_{1}=\kappa^{2}\left(1-\rho^{2}\right) / 2$. Thus, by taking $a=0$ and $b=b_{1}$ in (3.30)-(3.31) with $r_{0}$ replaced by $v_{0}$, we obtain

$$
\mathbb{E} \exp \left\{\frac{\kappa^{2}\left(1-\rho^{2}\right)}{2} \int_{0}^{t} v_{s} \mathrm{~d} s\right\}=M_{2}\left(0, \frac{\kappa^{2}\left(1-\rho^{2}\right)}{2}, t\right)=\hat{C}_{1}(\kappa, t) \mathrm{e}^{\hat{C}_{2}(\kappa, t) \nu_{0}}
$$

where

$$
\begin{aligned}
\hat{\Delta}(\kappa) & =\sqrt{\gamma^{2}-\kappa^{2} \delta^{2}\left(1-\rho^{2}\right)} \\
\hat{C}_{1}(\kappa, t) & =\left(\frac{\mathrm{e}^{\gamma t / 2}}{\cosh (\hat{\Delta}(\kappa) t / 2)+\gamma \sinh (\hat{\Delta}(\kappa) t / 2) / \hat{\Delta}(\kappa)}\right)^{2 \gamma \iota / \delta^{2}} \\
\hat{C}_{2}(\kappa, t) & =\frac{\kappa^{2}\left(1-\rho^{2}\right)}{\gamma+\hat{\Delta}(\kappa) \operatorname{coth}(\hat{\Delta}(\kappa) t / 2)}
\end{aligned}
$$

Therefore,

$$
\mathbb{E} \exp \left\{\frac{\kappa^{2}\left(1-\rho^{2}\right)}{2} \int_{0}^{t} v_{s} \mathrm{~d} s\right\}<\infty
$$

for the $\kappa$ as specified in (3.48). Applying Girsanov's theorem to $K_{1}(t)$ in (3.49) gives

$$
\begin{aligned}
K_{1}(t) & =\mathbb{E}\left(\exp \left\{-\kappa \sqrt{1-\rho^{2}} \int_{0}^{t} \sqrt{v_{s}} \mathrm{~d} W_{s}^{(3)}\right\} \mid W_{s}^{(2)}, 0 \leq s \leq t\right) \\
& =\exp \left\{\frac{\kappa^{2}\left(1-\rho^{2}\right)}{2} \int_{0}^{t} v_{s} \mathrm{~d} s\right\} .
\end{aligned}
$$


Substituting (3.50) into (3.49) and using (3.37), we obtain

$$
\begin{aligned}
\mathbb{E} Z_{t}^{-\kappa}= & \mathrm{e}^{-\kappa \mu t} \mathbb{E} \exp \left\{\left(\frac{\kappa^{2}\left(1-\rho^{2}\right)}{2}+\frac{\kappa}{2}\right) \int_{0}^{t} v_{s} \mathrm{~d} s-\kappa \rho \int_{0}^{t} \sqrt{v_{s}} \mathrm{~d} W_{s}^{(2)}\right\} \\
= & \exp \left\{-\kappa \mu t+\frac{\kappa \rho v_{0}}{\delta}+\frac{\kappa \rho \gamma \iota t}{\delta}\right\} \\
& \times \mathbb{E} \exp \left\{-\frac{\kappa \rho}{\delta} v_{t}+\left(\frac{\kappa^{2}\left(1-\rho^{2}\right)}{2}+\frac{\kappa}{2}-\frac{\kappa \rho \gamma}{\delta}\right) \int_{0}^{t} v_{s} \mathrm{~d} s\right\} \\
= & \exp \left\{-\kappa \mu t+\frac{\kappa \rho v_{0}}{\delta}+\frac{\kappa \rho \gamma \iota t}{\delta}\right\} M_{2}\left(-\frac{\kappa \rho}{\delta}, \frac{\kappa^{2}\left(1-\rho^{2}\right)}{2}+\frac{\kappa}{2}-\frac{\kappa \rho \gamma}{\delta}, t\right) .
\end{aligned}
$$

From (3.48), we see that $\gamma^{2}>2 \delta^{2} b_{2}$ when $b_{2}=\kappa^{2}\left(1-\rho^{2}\right) / 2+\kappa / 2-\kappa \rho \gamma / \delta$. Then, $M_{2}(a, b, t)$ exists when $a=-\kappa \rho / \delta$ and $b=b_{2}$. Thus, by (3.30)-(3.31) with $r_{0}$ replaced by $v_{0}$, we have

$$
\mathbb{E} Z_{t}^{-\kappa}=C_{1}(\kappa, t) \mathrm{e}^{-\kappa \mu t+C_{2}(\kappa, t) v_{0}},
$$

where

$$
\begin{aligned}
\Delta(\kappa) & =\sqrt{(\gamma+\kappa \rho \delta)^{2}-\delta^{2}\left(\kappa^{2}+\kappa\right)}, \\
C_{1}(\kappa, t) & =\left(\frac{\mathrm{e}^{(\gamma+\kappa \rho \delta) t / 2}}{\cosh (\Delta(\kappa) t / 2)+(\gamma+\kappa \rho \delta) \sinh (\Delta(\kappa) t / 2) / \Delta(\kappa)}\right)^{2 \gamma \iota / \delta^{2}}, \\
C_{2}(\kappa, t) & =\frac{\kappa^{2}+\kappa}{(\gamma+\kappa \rho \delta)+\Delta(\kappa) \operatorname{coth}(\Delta(\kappa) t / 2)} .
\end{aligned}
$$

It follows that $\int_{0}^{t} \mathbb{E} Z_{s}^{-\kappa} \mathrm{d} s<\infty$ for the $\kappa$ as specified in (3.48). By Theorem 2.1 and (3.52)(3.55), we obtain (3.41).

Next, we prove relation (3.46). From (3.40) and (3.45), we can take some $\kappa>\alpha^{\prime}=$ $\max \{\alpha, 2\}$ such that (3.48) and

$$
-\kappa \mu+\frac{\gamma \iota}{\delta^{2}}\left((\gamma+\kappa \rho \delta)-\sqrt{(\gamma+\kappa \rho \delta)^{2}-\delta^{2} \kappa(\kappa+1)}\right)<0
$$

hold simultaneously. For the fixed $\kappa$, by (3.52)-(3.55), we have

$$
\mathbb{E} Z_{t}^{-\kappa} \sim \widetilde{C}_{0} \exp \left\{-\kappa \mu t+\frac{\gamma \iota t}{\delta^{2}}\left((\gamma+\kappa \rho \delta)-\sqrt{(\gamma+\kappa \rho \delta)^{2}-\delta^{2} \kappa(\kappa+1)}\right)\right\} \quad \text { as } t \rightarrow \infty,
$$

where $\widetilde{C}_{0}$ is a positive constant. It follows that $\int_{0}^{\infty} \max \left\{s^{\eta}, 1\right\} \mathbb{E} Z_{s}^{-\kappa} \mathrm{d} s<\infty$ for the fixed $\kappa$ and any $\eta>0$. Thus, by Theorem 2.2 and (3.52)-(3.55), we obtain (3.46).

Remark 3.2. Drăgulescu and Yakovenko [10] derived the explicit expression for the probability density function of the Heston process $\left\{Z_{t}, t \geq 0\right\}$ defined in (3.36)-(3.37) and compared it with financial data. Using the Dow Jones Industrial Index data for the 20-year period 19822001, they obtained the fitting parameters $\gamma=11.35, \iota=0.022, \delta=0.618$, and $\mu=0.143$ in the unit of $1 /$ year when $\rho=0$, and found an excellent agreement between the expression and the Dow Jones data. For the four fitting parameters, the conditions in Theorem 3.4 can be satisfied over a certain range of $\alpha$ when $\rho=0$. Indeed, $2 \gamma \iota / \delta^{2} \approx 1.3$; if $\alpha^{\prime}=\max \{\alpha, 2\} \leq 17.87$ then $\gamma>\delta \sqrt{\alpha^{\prime}\left(\alpha^{\prime}+1\right)}$; and if $\alpha^{\prime}<10.66$, then $\gamma \iota\left(\gamma-\sqrt{\gamma^{2}-\delta^{2} \alpha^{\prime}\left(\alpha^{\prime}+1\right)}\right)<\alpha^{\prime} \delta^{2} \mu$. Thus, for the case $0<\alpha<10.66$, all the conditions in Theorem 3.4 are satisfied. 
Remark 3.3. Theorem 3.4 assumes that the variance process $\left\{v_{t}, t \geq 0\right\}$ in (3.37) starts from a positive constant $\nu_{0}$. In practice, however, this constant cannot be determined directly by financial data through numerical methods. It is more reasonable to assume that $v_{0}$ is a positive $\mathrm{RV}$ distributed by the stationary distribution function of the variance process $\left\{v_{t}, t \geq 0\right\}$.

Let $\Pi_{t}\left(v \mid v_{i}\right)$ be the probability density function of $v_{t}$ which has variance $v$ at time $t$ given the initial variance $v_{i}$ at time 0 . Then, $\Pi_{t}\left(v \mid v_{i}\right)$ determines the evolution of $\left\{v_{t}, t \geq 0\right\}$ and satisfies the well-known forward Kolmogorov equation

$$
\frac{\partial}{\partial t} \Pi_{t}\left(v \mid v_{i}\right)=\frac{\partial}{\partial v}\left[\gamma(v-\iota) \Pi_{t}\left(v \mid v_{i}\right)\right]+\frac{\delta^{2}}{2} \frac{\partial^{2}}{\partial v^{2}}\left[v \Pi_{t}\left(v \mid v_{i}\right)\right] .
$$

Equation (3.56) has the stationary solution (without relation to $v_{i}$ )

$$
\Pi_{*}(v)=\frac{a^{a}}{\Gamma(a)} \frac{v^{a-1}}{\iota^{a}} \mathrm{e}^{-v a / \iota}, \quad a=\frac{2 \gamma \iota}{\delta^{2}},
$$

which is a gamma density function.

Let $v_{0}$ be a positive RV distributed by $\Pi_{*}$. The moment generating function of $v_{0}$ is

$$
M_{3}(u)=\mathbb{E} \mathrm{e}^{u \nu_{0}}=\left(\frac{2 \gamma}{2 \gamma-\delta^{2} u}\right)^{2 \gamma \iota / \delta^{2}}
$$

provided that $2 \gamma>\delta^{2} u$. In this case, we have the following theorem.

Theorem 3.5. Under the conditions of Theorem 3.4, if $\nu_{0}$ is a positive RV distributed by $\Pi_{*}$ in (3.57) and the constant $\alpha^{\prime}=\max \{\alpha, 2\}$ satisfies (3.40), then, for arbitrarily fixed $t>0$,

$$
\Psi(x, t) \sim \lambda \bar{F}(x) \int_{0}^{t} C_{1}(\alpha, s) M_{3}\left(C_{2}(\alpha, s)\right) \mathrm{e}^{-\mu \alpha s} \mathrm{~d} s \quad \text { as } x \rightarrow \infty,
$$

where $C_{1}(\alpha, s)$ and $C_{2}(\alpha, s)$ are defined in (3.42)-(3.44), and $M_{3}(\cdot)$ is defined in (3.58). Furthermore, if the constant $\alpha^{\prime}$ also satisfies (3.45) then

$$
\Psi(x) \sim \lambda \bar{F}(x) \int_{0}^{\infty} C_{1}(\alpha, s) M_{3}\left(C_{2}(\alpha, s)\right) \mathrm{e}^{-\mu \alpha s} \mathrm{~d} s \quad \text { as } x \rightarrow \infty .
$$

Proof. Following the derivations in (3.47)-(3.55) and further conditioning on $v_{0}$ in (3.51) and (3.52), we obtain

$$
\mathbb{E} Z_{t}^{-\kappa}=\mathbb{E} \mathrm{e}^{-\kappa \xi_{t}}=C_{1}(\kappa, t) \mathrm{e}^{-\kappa \mu t} \mathbb{E} \mathrm{e}^{C_{2}(\kappa, t) \nu_{0}}=C_{1}(\kappa, t) \mathrm{e}^{-\kappa \mu t} M_{3}\left(C_{2}(\kappa, t)\right),
$$

with $\kappa$ specified in (3.48), $C_{1}(\kappa, t)$ and $C_{2}(\kappa, t)$ defined in (3.53)-(3.55), and $M_{3}(\cdot)$ defined in (3.58). Here we need to show that $M_{3}(u)$ exists when $u=C_{2}(\kappa, t)$. Indeed, by $\gamma+\kappa \rho \delta-$ $\delta \sqrt{\kappa(\kappa+1)}>0$ (see (3.48)) and $\rho \leq 0$, we have $\gamma+\kappa \rho \delta>0$ and $2 \gamma>\gamma+\kappa \rho \delta$. Hence, $2 \gamma(\gamma+\kappa \rho \delta)>(\gamma+\kappa \rho \delta)^{2}>\delta^{2} \kappa(\kappa+1)$, from which we obtain

$$
C_{2}(\kappa, t)=\frac{\kappa^{2}+\kappa}{(\gamma+\kappa \rho \delta)+\Delta(\kappa) \operatorname{coth}(\Delta(\kappa) t / 2)}<\frac{\kappa^{2}+\kappa}{\gamma+\kappa \rho \delta}<\frac{2 \gamma}{\delta^{2}} .
$$

Therefore, $M_{3}(u)$ exists when $u=C_{2}(\kappa, t)$. Following the discussions after (3.55) in the proof of Theorem 3.4, we conclude the proof. 


\section{Lemmas}

To prove the main results in this paper, we need a series of preliminaries. In the sequel, $C$ always represents a positive constant which may vary in different places. For a distribution function $F \in \mathcal{C}$, it is well known that $F$ is long tailed, namely, for any $l>0$,

$$
\bar{F}(x+l) \sim \bar{F}(x) .
$$

For a distribution function $F \in \mathcal{C}$, from Proposition 2.2.1 of [2], we know that, for any $p>\mathbb{J}_{F}^{+}$, there exist positive constants $C_{p}$ and $D_{p}$ such that

$$
\frac{\bar{F}(y)}{\bar{F}(x)} \leq C_{p}\left(\frac{x}{y}\right)^{p}
$$

holds uniformly for all $x \geq y \geq D_{p}$. Fixing the variable $y$ in (4.2) leads to

$$
x^{-p}=o(\bar{F}(x)) \quad \text { for any } p>\mathbb{J}_{F}^{+} .
$$

Lemma 4.1. Suppose that $X$ is a nonnegative $R V$ with distribution function $F \in \mathcal{C}$ and that $\Theta$ is another nonnegative $R V$ independent of $X$. Then, we have the following three results.

(i) For arbitrarily fixed $\delta_{1}>0$ and $p>\mathbb{J}_{F}^{+}$, there exists some positive constant $C$ without relation to $\delta_{1}$ and $\Theta$ such that, for all large $x$,

$$
\mathbb{P}\left(X \Theta>\delta_{1} x \mid \Theta\right) \leq C \bar{F}(x)\left(\delta_{1}^{-p} \Theta^{p} \mathbf{1}_{\left[\Theta>\delta_{1}\right]}+\mathbf{1}_{\left[\Theta \leq \delta_{1}\right]}\right) .
$$

(ii) If $\mathbb{J}_{F}^{-}>0$ then, for arbitrarily fixed $\delta_{1}>0$ and $0<p_{1}<\mathbb{J}_{F}^{-} \leq \mathbb{J}_{F}^{+}<p_{2}<\infty$, there exists some positive constant $C$ without relation to $\delta_{1}$ and $\Theta$ such that, for all large $x$,

$$
\mathbb{P}\left(X \Theta>\delta_{1} x \mid \Theta\right) \leq C \bar{F}(x)\left(\delta_{1}^{-p_{1}} \Theta^{p_{1}}+\delta_{1}^{-p_{2}} \Theta^{p_{2}}\right) .
$$

(iii) If $\Theta$ is not degenerate at 0 and $\int_{0}^{\infty} x^{p} \mathbb{P}(\Theta \in \mathrm{d} x)<\infty$ for some $p>\mathbb{J}_{F}^{+}$, then $X \Theta \in \mathcal{C}$ and

$$
\mathbb{P}(X \Theta>x) \asymp \bar{F}(x) .
$$

Proof. Result (i) is a direct consequence of Lemma 3.2 of [16] with some obvious modifications. Result (ii) is from Lemma 4.1.5 of [30] with some adjustment. Result (iii) is the combination of Theorem 3.3(iv) of [8] and Lemma 2.5 of [30].

The proofs of the following two lemmas are inspired by those of Lemmas 2.2 and 2.3 of [31], respectively.

Lemma 4.2. Suppose that $X_{1}$ and $X_{2}$ are two identically distributed RVs with common distribution function $F \in \mathcal{C}$, and that $\Theta_{1}$ and $\Theta_{2}$ are another two positive and bounded $R V s$ independent of $\left(X_{1}, X_{2}\right)$. If $X_{1}$ and $X_{2}$ are bivariate upper tail independent, i.e. they satisfy relation (2.2), then

$$
\lim _{x \rightarrow \infty} \frac{\mathbb{P}\left(X_{1} \Theta_{1}>x, X_{2} \Theta_{2}>x\right)}{\mathbb{P}\left(X_{k} \Theta_{k}>x\right)}=0, \quad k=1,2 .
$$


Proof. Without loss of generality, we assume that both $\Theta_{1}$ and $\Theta_{2}$ are bounded from above by the same constant $b>1$. Denote the distribution function of $\Theta_{i}$ by $H_{i}, i=1,2$, and the joint distribution function of $\left(\Theta_{1}, \Theta_{2}\right)$ by $H$. Clearly,

$$
\begin{aligned}
& \mathbb{P}\left(X_{1} \Theta_{1}>x, X_{2} \Theta_{2}>x\right) \\
&=\left(\iint_{0<u \leq v \leq b}+\iint_{0<v<u \leq b}\right) \mathbb{P}\left(X_{1}>\frac{x}{u}, X_{2}>\frac{x}{v}\right) \mathrm{d} H(u, v) \\
& \leq \iint_{0<u \leq v \leq b} \mathbb{P}\left(X_{1}>\frac{x}{v}, X_{2}>\frac{x}{v}\right) \mathrm{d} H(u, v) \\
&+\iint_{0<v<u \leq b} \mathbb{P}\left(X_{1}>\frac{x}{u}, X_{2}>\frac{x}{u}\right) \mathrm{d} H(u, v) \\
& \leq \int_{(0, b]} \mathbb{P}\left(X_{1}>\frac{x}{v}, X_{2}>\frac{x}{v}\right) \mathrm{d} H_{2}(v)+\int_{(0, b]} \mathbb{P}\left(X_{1}>\frac{x}{u}, X_{2}>\frac{x}{u}\right) \mathrm{d} H_{1}(u) \\
&=: \Delta_{1}(x)+\Delta_{2}(x) .
\end{aligned}
$$

From inequality (4.2), we know that, for some fixed $p>\mathbb{J}_{F}^{+}$, the inequalities

$$
\frac{\mathbb{P}\left(X_{k}>x / v\right)}{\mathbb{P}\left(X_{k}>x\right)} \leq C_{p} v^{p}, \quad k=1,2,
$$

hold uniformly for all $v \in(1, b]$ and all $x>b D_{p}$. For arbitrarily fixed $\varepsilon>0$, by relation (2.2), there exists some $x_{0}>b D_{p}$ such that, for all $x>x_{0}$,

$$
\sup _{0<v \leq b} \frac{\mathbb{P}\left(X_{1}>x / v, X_{2}>x / v\right)}{\mathbb{P}\left(X_{k}>x / v\right)} \leq \varepsilon, \quad k=1,2 .
$$

Thus, by (4.5)-(4.6), we can derive, for all $x>x_{0}$,

$$
\begin{aligned}
\frac{\Delta_{1}(x)}{\mathbb{P}\left(X_{k}>x\right)}= & \int_{(0,1]} \frac{\mathbb{P}\left(X_{1}>x / v, X_{2}>x / v\right)}{\mathbb{P}\left(X_{k}>x / v\right)} \frac{\mathbb{P}\left(X_{k}>x / v\right)}{\mathbb{P}\left(X_{k}>x\right)} \mathrm{d} H_{2}(v) \\
& +\int_{(1, b]} \frac{\mathbb{P}\left(X_{1}>x / v, X_{2}>x / v\right)}{\mathbb{P}\left(X_{k}>x / v\right)} \frac{\mathbb{P}>v)}{\mathbb{P}\left(X_{k}>x\right)} \mathrm{d} H_{2}(v) \\
\leq & \int_{(0,1]} \frac{\mathbb{P}\left(X_{1}>x / v, X_{2}>x / v\right)}{\mathbb{P}\left(X_{k}>x / v\right)} \mathrm{d} H_{2}(v) \\
& +C_{p} \int_{(1, b]} \frac{\mathbb{P}\left(X_{1}>x / v, X_{2}>x / v\right)}{\mathbb{P}\left(X_{k}>x / v\right)} v^{p} \mathrm{~d} H_{2}(v) \\
\leq & \sup _{0<v \leq b} \frac{\mathbb{P}\left(X_{1}>x / v, X_{2}>x / v\right)}{\mathbb{P}\left(X_{k}>x / v\right)}\left(H_{2}(1)-H_{2}(0)+C_{p} \int_{(1, b]} v^{p} \mathrm{~d} H_{2}(v)\right) \\
\leq & C \varepsilon, \quad k=1,2 .
\end{aligned}
$$

By Lemma 4.1(iii), we can take $x_{1}>x_{0}$ and $C>0$ such that, for all $x>x_{1}$,

$$
\mathbb{P}\left(X_{k}>x\right) \leq C \mathbb{P}\left(X_{k} \Theta_{k}>x\right), \quad k=1,2 .
$$

Hence, for all $x>x_{1}$,

$$
\frac{\Delta_{1}(x)}{\mathbb{P}\left(X_{k} \Theta_{k}>x\right)}=\frac{\Delta_{1}(x)}{\mathbb{P}\left(X_{k}>x\right)} \frac{\mathbb{P}\left(X_{k}>x\right)}{\mathbb{P}\left(X_{k} \Theta_{k}>x\right)} \leq C \varepsilon, \quad k=1,2 .
$$


Using the symmetry between $\Delta_{1}(x)$ and $\Delta_{2}(x)$, we find that there exist $x_{2}>x_{1}$ and $C>0$ such that, for all $x>x_{2}$,

$$
\frac{\Delta_{2}(x)}{\mathbb{P}\left(X_{k} \Theta_{k}>x\right)} \leq C \varepsilon, \quad k=1,2 .
$$

Combining (4.7) and (4.8) with (4.4), we have, for all $x>x_{2}$,

$$
\frac{\mathbb{P}\left(X_{1} \Theta_{1}>x, X_{2} \Theta_{2}>x\right)}{\mathbb{P}\left(X_{k} \Theta_{k}>x\right)} \leq C \varepsilon, \quad k=1,2 .
$$

By the arbitrariness of $\varepsilon>0$, we conclude the proof.

Lemma 4.3. Suppose that $\left\{X_{i}, 1 \leq i \leq n\right\}$ is a sequence of nonnegative and identically distributed $R V s$ with common distribution function $F \in \mathcal{C}$, satisfying relation (2.2), while $\left\{\Theta_{i}, 1 \leq i \leq n\right\}$ is a sequence of bounded, nonnegative, and not-degenerate-at-zero $R V s$ independent of $\left\{X_{i}, 1 \leq i \leq n\right\}$. Then

$$
\mathbb{P}\left(\sum_{i=1}^{n} X_{i} \Theta_{i}>x\right) \sim \sum_{i=1}^{n} \mathbb{P}\left(X_{i} \Theta_{i}>x\right) .
$$

Proof. Clearly, (4.9) holds for $n=1$. Hence, we suppose that $n \geq 2$. Note that

$$
\begin{aligned}
\mathbb{P}\left(\sum_{i=1}^{n} X_{i} \Theta_{i}>x\right) & \geq \mathbb{P}\left(\bigcup_{i=1}^{n}\left(X_{i} \Theta_{i}>x\right)\right) \\
& \geq \sum_{i=1}^{n} \mathbb{P}\left(X_{i} \Theta_{i}>x\right)-\sum_{1 \leq i, j \leq n, i \neq j} \mathbb{P}\left(X_{i} \Theta_{i}>x, X_{j} \Theta_{j}>x\right) .
\end{aligned}
$$

Note that $\left\{X_{k}, \Theta_{k}, 1 \leq k \leq n\right\}$ are on the probability space $(\Omega, \mathcal{F}, \mathbb{P})$. For any $1 \leq i, j \leq n$, $i \neq j$, define $B_{i j}=\left\{\Theta_{i}>0, \Theta_{j}>0\right\}$. For the case $\mathbb{P}\left(B_{i j}\right)>0$, let $\left(\Omega \cap B_{i j}, \mathcal{F} \cap B_{i j}, \mathbb{P}_{B_{i j}}\right)$ be the trace of $(\Omega, \mathcal{F}, \mathbb{P})$ on the set $B_{i j}$, where $\mathcal{F} \cap B_{i j}=\left\{F \cap B_{i j}: F \in \mathcal{F}\right\}$ and, for any $A \in \mathcal{F}$,

$$
\mathbb{P}_{B_{i j}}\left(A \cap B_{i j}\right)=\frac{\mathbb{P}\left(A \cap B_{i j}\right)}{\mathbb{P}\left(B_{i j}\right)}=\mathbb{P}\left(A \mid B_{i j}\right) .
$$

Clearly, $\left(\Omega \cap B_{i j}, \mathcal{F} \cap B_{i j}, \mathbb{P}_{B_{i j}}\right)$ is a probability space. From the independence between $\left(X_{i}, X_{j}\right)$ and $\left(\Theta_{i}, \Theta_{j}\right)$ on $(\Omega, \mathcal{F}, \mathbb{P})$, we can verify that all the assumptions on $\left\{X_{k}, \Theta_{k}\right.$, $k=i, j\}$ in this lemma are preserved on this new probability space. In addition, $\Theta_{i}$ and $\Theta_{j}$ are also positive on this new probability space. Thus, for arbitrarily fixed $\varepsilon, a>0$, by Lemma 4.2, there exists some $x_{i j}(a)>0$ such that, for all $x>x_{i j}(a)$,

$$
\begin{aligned}
\mathbb{P}\left(X_{i} \Theta_{i}>a x, X_{j} \Theta_{j}>a x\right) \\
\quad=\mathbb{P}\left(X_{i} \Theta_{i}>a x, X_{j} \Theta_{j}>a x, \Theta_{i}>0, \Theta_{j}>0\right) \\
\quad=\mathbb{P}\left(X_{i} \Theta_{i}>a x, X_{j} \Theta_{j}>a x \mid \Theta_{i}>0, \Theta_{j}>0\right) \mathbb{P}\left(\Theta_{i}>0, \Theta_{j}>0\right) \\
\quad \leq \varepsilon \mathbb{P}\left(X_{i} \Theta_{i}>a x \mid \Theta_{i}>0, \Theta_{j}>0\right) \mathbb{P}\left(\Theta_{i}>0, \Theta_{j}>0\right) \\
\quad=\varepsilon \mathbb{P}\left(X_{i} \Theta_{i}>a x, \Theta_{i}>0, \Theta_{j}>0\right) \\
\quad \leq \varepsilon \mathbb{P}\left(X_{i} \Theta_{i}>a x\right) .
\end{aligned}
$$


For the case $\mathbb{P}\left(B_{i j}\right)=0$, it is trivial to show that, for all $x>0$ and $a>0$,

$$
\begin{aligned}
\mathbb{P}\left(X_{i} \Theta_{i}>a x, X_{j} \Theta_{j}>a x\right) & =\mathbb{P}\left(X_{i} \Theta_{i}>a x, X_{j} \Theta_{j}>a x, \Theta_{i}>0, \Theta_{j}>0\right) \\
& =0 \\
& \leq \varepsilon \mathbb{P}\left(X_{i} \Theta_{i}>a x\right) .
\end{aligned}
$$

Hence, by taking $a=1$ in (4.11) and (4.12), we obtain, for all $x>x_{0}=\max _{1 \leq i, j \leq n, i \neq j}\left\{x_{i j}(1)\right\}$,

$$
\sum_{1 \leq i, j \leq n, i \neq j} \mathbb{P}\left(X_{i} \Theta_{i}>x, X_{j} \Theta_{j}>x\right) \leq(n-1) \varepsilon \sum_{i=1}^{n} \mathbb{P}\left(X_{i} \Theta_{i}>x\right) .
$$

Substituting (4.13) into (4.10), we obtain, for all $x>x_{0}$,

$$
\mathbb{P}\left(\sum_{i=1}^{n} X_{i} \Theta_{i}>x\right) \geq(1-C \varepsilon) \sum_{i=1}^{n} \mathbb{P}\left(X_{i} \Theta_{i}>x\right) .
$$

Next we prove the upper-bound version of relation (4.9). According to Lemma 4.1(iii), the distribution function $G_{i}$ of $X_{i} \Theta_{i}$ belongs to the class $\mathcal{C}$ for any $1 \leq i \leq n$. Then, for arbitrarily fixed $\varepsilon>0$, by the definition of $\mathcal{C}$ in $(2.1)$, we can take $v_{0} \in\left(\frac{1}{2}, 1\right)$ and $x_{1}>x_{0}$ such that, for all $x>x_{1}$,

$$
\sum_{i=1}^{n} \mathbb{P}\left(X_{i} \Theta_{i}>v_{0} x\right) \leq(1+\varepsilon) \sum_{i=1}^{n} \mathbb{P}\left(X_{i} \Theta_{i}>x\right) .
$$

For the fixed $v_{0}$, we have

$$
\begin{aligned}
\mathbb{P}\left(\sum_{i=1}^{n} X_{i} \Theta_{i}>x\right) \leq & \mathbb{P}\left(\bigcup_{i=1}^{n}\left(X_{i} \Theta_{i}>v_{0} x\right)\right) \\
& +\mathbb{P}\left(\sum_{i=1}^{n} X_{i} \Theta_{i}>x, \bigcap_{j=1}^{n}\left(X_{j} \Theta_{j} \leq v_{0} x\right)\right) \\
= & : I_{1}+I_{2} .
\end{aligned}
$$

For $I_{1}$, by inequality (4.15), we obtain, for all $x>x_{1}$,

$$
I_{1} \leq \sum_{i=1}^{n} \mathbb{P}\left(X_{i} \Theta_{i}>v_{0} x\right) \leq(1+\varepsilon) \sum_{i=1}^{n} \mathbb{P}\left(X_{i} \Theta_{i}>x\right) .
$$

For $I_{2}$, we have

$$
\begin{aligned}
I_{2} & =\mathbb{P}\left(\sum_{i=1}^{n} X_{i} \Theta_{i}>x, \bigcap_{j=1}^{n}\left(X_{j} \Theta_{j} \leq v_{0} x\right), \max _{1 \leq k \leq n} X_{k} \Theta_{k}>\frac{x}{n}\right) \\
& \leq \sum_{k=1}^{n} \mathbb{P}\left(\sum_{i=1}^{n} X_{i} \Theta_{i}>x, X_{k} \Theta_{k} \leq v_{0} x, X_{k} \Theta_{k}>\frac{x}{n}\right) \\
& \leq \sum_{k=1}^{n} \mathbb{P}\left(\sum_{i=1, i \neq k}^{n} X_{i} \Theta_{i}>\left(1-v_{0}\right) x, X_{k} \Theta_{k}>\frac{x}{n}\right) \\
& \leq \sum_{1 \leq i, k \leq n, i \neq k} \mathbb{P}\left(X_{i} \Theta_{i}>\frac{\left(1-v_{0}\right) x}{n-1}, X_{k} \Theta_{k}>\frac{x}{n}\right) .
\end{aligned}
$$


Define $a_{0}=\left(1-v_{0}\right) /(n-1)$. Since $v_{0} \in\left(\frac{1}{2}, 1\right)$ and $n \geq 2$, we have $0<a_{0}<1 / n<1$. For all $1 \leq i, k \leq n, i \neq k$, and the fixed $\varepsilon>0$ as before, by taking $a=a_{0}$ in (4.11)-(4.12), we obtain, for all $x>x_{i k}\left(a_{0}\right)$,

$$
\mathbb{P}\left(X_{i} \Theta_{i}>a_{0} x, X_{k} \Theta_{k}>a_{0} x\right) \leq \varepsilon \mathbb{P}\left(X_{i} \Theta_{i}>a_{0} x\right) .
$$

Recall that, by Lemma 4.1(iii), the distribution function $G_{i}$ of $X_{i} \Theta_{i}$ belongs to the class $\mathcal{C}$ for any $1 \leq i \leq n$. Then, for some fixed $p>\max _{1 \leq i \leq n}\left\{\mathbb{J}_{G_{i}}^{+}\right\}$, by inequality (4.2), there exist positive constants $C_{p}$ and $D_{p}$ such that, for all $x>D_{p}$ and all $1 \leq i \leq n$,

$$
\mathbb{P}\left(X_{i} \Theta_{i}>a_{0} x\right) \leq C_{p} a_{0}^{-p} \mathbb{P}\left(X_{i} \Theta_{i}>x\right) .
$$

Hence, by (4.18)-(4.20) we obtain, for all $x>x_{2}=\max _{1 \leq i, k \leq n, i \neq k}\left\{x_{i k}\left(a_{0}\right), D_{p}\right\}$,

$$
\begin{aligned}
I_{2} & \leq \sum_{1 \leq i, k \leq n, i \neq k} \mathbb{P}\left(X_{i} \Theta_{i}>a_{0} x, X_{k} \Theta_{k}>a_{0} x\right) \\
& \leq(n-1) \varepsilon \sum_{i=1}^{n} \mathbb{P}\left(X_{i} \Theta_{i}>a_{0} x\right) \\
& \leq(n-1) C_{p} a_{0}^{-p} \varepsilon \sum_{i=1}^{n} \mathbb{P}\left(X_{i} \Theta_{i}>x\right) .
\end{aligned}
$$

Substituting (4.17) and (4.21) into (4.16), we obtain, for all $x>x_{2}$,

$$
\mathbb{P}\left(\sum_{i=1}^{n} X_{i} \Theta_{i}>x\right) \leq(1+C \varepsilon) \sum_{i=1}^{n} \mathbb{P}\left(X_{i} \Theta_{i}>x\right) .
$$

Therefore, inequalities (4.14) and (4.22) hold for all $x>x_{2}$. By the arbitrariness of $\varepsilon>0$, we conclude the proof.

\section{Proofs of the theorems}

Clearly, $U_{t}$ in (1.1) is equivalent to

$$
U_{t}=\mathrm{e}^{\xi_{t}}\left(x-\sum_{n=1}^{\infty} X_{n} \mathrm{e}^{-\xi_{\tau_{n}-}} \mathbf{1}_{\left[\tau_{n} \leq t\right]}+c \int_{0}^{t} \mathrm{e}^{-\xi_{s}} \mathrm{~d} s+\sigma \int_{0}^{t} \mathrm{e}^{-\xi_{s}} \mathrm{~d} B_{s}\right) .
$$

For notational convenience, define

$$
\vartheta_{n}(t)=\mathrm{e}^{-\xi_{\tau_{n}-}} \mathbf{1}_{\left[\tau_{n} \leq t\right]}, \quad n \geq 1 .
$$

Then, the ruin probability up to time $t>0$ and the infinite-time ruin probability can be respectively rewritten as

$$
\Psi(x, t)=\mathbb{P}\left(\sup _{0<s \leq t}\left\{\sum_{n=1}^{\infty} X_{n} \vartheta_{n}(s)-c \int_{0}^{s} \mathrm{e}^{-\xi_{v}} \mathrm{~d} v-\sigma \int_{0}^{s} \mathrm{e}^{-\xi_{v}} \mathrm{~d} B_{v}\right\}>x\right)
$$

and

$$
\Psi(x)=\mathbb{P}\left(\sup _{0<s<\infty}\left\{\sum_{n=1}^{\infty} X_{n} \vartheta_{n}(s)-c \int_{0}^{s} \mathrm{e}^{-\xi_{v}} \mathrm{~d} v-\sigma \int_{0}^{s} \mathrm{e}^{-\xi_{v}} \mathrm{~d} B_{v}\right\}>x\right) .
$$


Proof of Theorem 2.1. We first prove the upper-bound version of relation (2.3). For any $q \geq 0$, we have

$$
\sum_{n=1}^{\infty} n^{q} \frac{s^{n-1} \lambda^{n}}{(n-1) !} \mathrm{e}^{-\lambda s} \leq \begin{cases}C \max \left\{s^{q}, 1\right\}, & q=0,1,2, \ldots \\ C \max \left\{s^{[q]+1}, 1\right\}, & \text { otherwise }\end{cases}
$$

where $[q]$ is the integer part of $q$. Note that $\tau_{n}$ is a gamma RV satisfying

$$
\mathbb{P}\left(\tau_{n} \in \mathrm{d} s\right)=\frac{s^{n-1} \lambda^{n}}{(n-1) !} \mathrm{e}^{-\lambda s} \mathrm{~d} s, \quad s \geq 0 .
$$

Then, by Hölder's inequality and Jensen's inequality, we can derive, for any $0<p \leq \kappa$ and $q \geq 0$

$$
\begin{array}{rl}
\sum_{n=1}^{\infty} n^{q} & \mathbb{E}\left(\vartheta_{n}^{p}(t)+\mathbf{1}_{\left[\vartheta_{n}(t) \leq 1\right]}\right) \\
= & \sum_{n=1}^{\infty} n^{q}\left(\int_{0}^{t} \mathbb{E} \mathrm{e}^{-p \xi_{s}-} \mathbb{P}\left(\tau_{n} \in \mathrm{d} s\right)+\int_{0}^{t} \mathbb{P}\left(\mathrm{e}^{-\xi_{s-}} \leq 1\right) \mathbb{P}\left(\tau_{n} \in \mathrm{d} s\right)\right) \\
& =\sum_{n=1}^{\infty} n^{q}\left(\int_{0}^{t} \mathbb{E} \mathrm{e}^{-p \xi_{s}} \frac{s^{n-1} \lambda^{n}}{(n-1) !} \mathrm{e}^{-\lambda s} \mathrm{~d} s+\int_{0}^{t} \mathbb{P}\left(\mathrm{e}^{-\xi_{s}} \leq 1\right) \frac{s^{n-1} \lambda^{n}}{(n-1) !} \mathrm{e}^{-\lambda s} \mathrm{~d} s\right) \\
& \leq C \max \left\{t^{[q]+1}, 1\right\}\left(\int_{0}^{t} \mathbb{E} \mathrm{e}^{-p \xi_{s}} \mathrm{~d} s+t\right) \\
& \leq C \int_{0}^{t} \mathbb{E}^{p / \kappa} \mathrm{e}^{-\kappa \xi_{s}} \mathrm{~d} s+C \\
& \leq C\left(\int_{0}^{t} \mathbb{E}^{-\kappa \xi_{s}} \mathrm{~d} s\right)^{p / \kappa}+C \\
& <\infty
\end{array}
$$

Take $\mathbb{J}_{F}^{+}<p \leq \kappa$. For arbitrarily fixed $\varepsilon>0$, by (5.4), we can find some integer $N>1$ such that

$$
\begin{gathered}
\sum_{n=N+1}^{\infty} n^{2 p} \mathbb{E}\left(\vartheta_{n}^{p}(t)+\mathbf{1}_{\left[\vartheta_{n}(t) \leq 1\right]}\right)<\varepsilon \\
\sum_{n=N+1}^{\infty} \frac{1}{n^{2}}<1 .
\end{gathered}
$$

Note that (5.4) implies that $\mathbb{E} \vartheta_{n}^{\kappa}(t)<\infty$ for any $n \geq 1$. According to Lemma 4.1(iii), $X_{n} \vartheta_{n}(t) \in \mathcal{C}$ for any $1 \leq n \leq N$. Then, by the definition of the class $\mathcal{C}$ in (2.1), we can show that there exist $v_{0} \in\left(\frac{1}{2}, 1\right)$ and $x_{1}>0$ such that, for all $x>x_{1}$,

$$
\bar{F}\left(v_{0} x\right) \leq(1+\varepsilon) \bar{F}(x) \text { and } \sum_{n=1}^{N} \mathbb{P}\left(X_{n} \vartheta_{n}(t)>v_{0} x\right) \leq(1+\varepsilon) \sum_{n=1}^{N} \mathbb{P}\left(X_{n} \vartheta_{n}(t)>x\right) .
$$


Define $D_{0, t}=\sup _{0 \leq s \leq t}\left|\int_{0}^{s} \mathrm{e}^{-\xi_{v}} \mathrm{~d} B_{v}\right|$. For the fixed $N$ and $v_{0}$, according to (5.1), we derive

$$
\begin{aligned}
\Psi(x, t) \leq & \mathbb{P}\left(\sum_{n=1}^{\infty} X_{n} \vartheta_{n}(t)+\sigma D_{0, t}>x\right) \\
\leq & \mathbb{P}\left(\sum_{n=1}^{N} X_{n} \vartheta_{n}(t)>v_{0} x\right)+\mathbb{P}\left(\sum_{n=N+1}^{\infty} X_{n} \vartheta_{n}(t)>\frac{1-v_{0}}{2} x\right) \\
& +\mathbb{P}\left(\sigma D_{0, t}>\frac{1-v_{0}}{2} x\right) \\
= & : I_{1}(t)+I_{2}(t)+I_{3}(t) .
\end{aligned}
$$

We first deal with $I_{1}(t)$. Note that $\mathbb{J}_{F}^{+}<p \leq \kappa$ and $\mathbb{E} \vartheta_{n}^{p}(t)<\infty$ for any $n \geq 1$. Then, we can choose $L>0$ such that, for all $1 \leq j, n \leq N$,

$$
\mathbb{E}\left(\vartheta_{n}^{p}(t) \mathbf{1}_{\left[\vartheta_{j}(t)>L\right]}\right)<\frac{\varepsilon}{N^{p+2}(1+\varepsilon)}, \quad \mathbb{P}\left(\vartheta_{j}(t)>L\right)<\frac{\varepsilon}{N^{2}(1+\varepsilon)} .
$$

Relying on this $L$, we can further split $I_{1}(t)$ into two parts as follows:

$$
\begin{aligned}
I_{1}(t)= & \mathbb{P}\left(\sum_{n=1}^{N} X_{n} \vartheta_{n}(t)>v_{0} x, \bigcap_{j=1}^{N}\left(\vartheta_{j}(t) \leq L\right)\right) \\
& +\mathbb{P}\left(\sum_{n=1}^{N} X_{n} \vartheta_{n}(t)>v_{0} x, \bigcup_{j=1}^{N}\left(\vartheta_{j}(t)>L\right)\right) \\
= & : I_{11}(t)+I_{12}(t) .
\end{aligned}
$$

For $I_{11}(t)$, by Lemma 4.3 and (5.7), we can find some $x_{2}>x_{1}$ such that, for all $x>x_{2}$,

$$
I_{11}(t) \leq(1+\varepsilon) \sum_{n=1}^{N} \mathbb{P}\left(X_{n} \vartheta_{n}(t)>v_{0} x\right) \leq(1+\varepsilon)^{2} \sum_{n=1}^{N} \mathbb{P}\left(X_{n} \vartheta_{n}(t)>x\right) .
$$

For $I_{12}(t)$, by Lemma 4.1(i), (5.7) and (5.9), we can find some $x_{3}>x_{2}$ such that, for all $x>x_{3}$,

$$
\begin{aligned}
& I_{12}(t) \leq \sum_{j=1}^{N} \mathbb{P}\left(\sum_{n=1}^{N} X_{n} \vartheta_{n}(t)>v_{0} x, \vartheta_{j}(t)>L\right) \\
& \leq \sum_{j=1}^{N} \sum_{n=1}^{N} \mathbb{P}\left(X_{n} \vartheta_{n}(t)>\frac{v_{0} x}{N}, \vartheta_{j}(t)>L\right) \\
& =\sum_{j=1}^{N} \sum_{n=1}^{N} \mathbb{E}\left(\mathbf{1}_{\left[\vartheta_{j}(t)>L\right]} \mathbb{P}\left(X_{n} \vartheta_{n}(t)>\frac{v_{0} x}{N} \mid \vartheta_{n}(t), \vartheta_{j}(t)\right)\right) \\
& \leq C \bar{F}\left(v_{0} x\right) \sum_{j=1}^{N} \sum_{n=1}^{N} \mathbb{E}\left(\mathbf{1}_{\left[\vartheta_{j}(t)>L\right]}\left(N^{p} \vartheta_{n}^{p}(t)+\mathbf{1}_{\left[\vartheta_{n}(t) \leq 1 / N\right]}\right)\right) \\
& \leq C(1+\varepsilon) \bar{F}(x) \sum_{j=1}^{N} \sum_{n=1}^{N}\left(N^{p} \mathbb{E}\left(\vartheta_{n}^{p}(t) \mathbf{1}_{\left[\vartheta_{j}(t)>L\right]}\right)+\mathbb{P}\left(\vartheta_{j}(t)>L\right)\right) \\
& \leq C \varepsilon \bar{F}(x) .
\end{aligned}
$$


Substituting (5.11)-(5.12) into (5.10), we have, for all $x>x_{3}$,

$$
I_{1}(t) \leq(1+C \varepsilon) \sum_{n=1}^{N} \mathbb{P}\left(X_{n} \vartheta_{n}(t)>x\right)+C \varepsilon \bar{F}(x) .
$$

For $I_{2}(t)$, by Lemma 4.1(i) and (5.5)-(5.6), we can take some $x_{4}>x_{3}$ such that, for all $x>x_{4}$,

$$
\begin{aligned}
I_{2}(t) & \leq \mathbb{P}\left(\sum_{n=N+1}^{\infty} X_{n} \vartheta_{n}(t)>\sum_{n=N+1}^{\infty} \frac{\left(1-v_{0}\right) x}{2 n^{2}}\right) \\
& \leq \sum_{n=N+1}^{\infty} \mathbb{P}\left(X_{n} \vartheta_{n}(t)>\frac{\left(1-v_{0}\right) x}{2 n^{2}}\right) \\
& =\sum_{n=N+1}^{\infty} \mathbb{E}\left(\mathbb{P}\left(X_{n} \vartheta_{n}(t)>\frac{\left(1-v_{0}\right) x}{2 n^{2}} \mid \vartheta_{n}(t)\right)\right) \\
& \leq C \bar{F}(x) \sum_{n=N+1}^{\infty}\left\{\left(\frac{1-v_{0}}{2}\right)^{-p} n^{2 p} \mathbb{E} \vartheta_{n}^{p}(t)+\mathbb{E} \mathbf{1}_{\left[\vartheta_{n}(t) \leq\left(1-v_{0}\right) / 2 n^{2}\right]}\right\} \\
& \leq C \bar{F}(x) \sum_{n=N+1}^{\infty} n^{2 p}\left(\mathbb{E} \vartheta_{n}^{p}(t)+\mathbb{E} \mathbf{1}_{\left[\vartheta_{n}(t) \leq 1\right]}\right) \\
& \leq C \varepsilon \bar{F}(x) .
\end{aligned}
$$

We next deal with $I_{3}(t)$. By the Burkholder-Davis-Gundy inequalities, Hölder's inequality, and Fubini's theorem, we obtain

$$
\begin{aligned}
\mathbb{E} D_{0, t}^{\kappa} & \leq C \mathbb{E}\left(\int_{0}^{t} \mathrm{e}^{-2 \xi_{s}} \mathrm{~d} s\right)^{\kappa / 2} \\
& \leq C t^{\kappa / 2-1} \mathbb{E} \int_{0}^{t} \mathrm{e}^{-\kappa \xi_{s}} \mathrm{~d} s \\
& =C t^{\kappa / 2-1} \int_{0}^{t} \mathbb{E} \mathrm{e}^{-\kappa \xi_{s}} \mathrm{~d} s \\
& <\infty
\end{aligned}
$$

According to relation (4.3), we can take some $x_{5}>x_{4}$ such that $x^{-\kappa} \leq \varepsilon \bar{F}(x)$ holds for all $x>x_{5}$. Hence, by Chebyshev's inequality and (5.15), we obtain, for all $x>x_{5}$,

$$
I_{3}(t) \leq\left(\frac{2 \sigma}{1-v_{0}}\right)^{\kappa} x^{-\kappa} \mathbb{E} D_{0, t}^{\kappa} \leq C \varepsilon \bar{F}(x)
$$

Recall that $\mathbb{E} \vartheta_{1}^{\kappa}(t)<\infty$. Then, by Lemma 4.1(iii), we can take some $x_{6}>x_{5}$ and $C>0$ such that, for all $x>x_{6}$,

$$
\bar{F}(x) \leq C \mathbb{P}\left(X_{1} \vartheta_{1}(t)>x\right) .
$$


Substituting (5.13)-(5.14) and (5.16) into (5.8) and using (5.3) and (5.17), we obtain, for all $x>x_{6}$,

$$
\begin{aligned}
\Psi(x, t) & \leq(1+C \varepsilon) \sum_{n=1}^{N} \mathbb{P}\left(X_{n} \vartheta_{n}(t)>x\right)+C \varepsilon \mathbb{P}\left(X_{1} \vartheta_{1}(t)>x\right) \\
& \leq(1+C \varepsilon) \sum_{n=1}^{\infty} \mathbb{P}\left(X_{n} \vartheta_{n}(t)>x\right) \\
& =(1+C \varepsilon) \sum_{n=1}^{\infty} \mathbb{P}\left(X_{n} \mathrm{e}^{-\xi_{\tau_{n}-}} \mathbf{1}_{\left[\tau_{n} \leq t\right]}>x\right) \\
& =(1+C \varepsilon) \sum_{n=1}^{\infty} \int_{0}^{t} \mathbb{P}\left(X_{1} \mathrm{e}^{-\xi_{s}}>x\right) \frac{s^{n-1} \lambda^{n}}{(n-1) !} \mathrm{e}^{-\lambda s} \mathrm{~d} s \\
& =(1+C \varepsilon) \lambda \int_{0}^{t} \mathbb{P}\left(X_{1} \mathrm{e}^{-\xi_{s}}>x\right) \mathrm{d} s .
\end{aligned}
$$

We next prove the lower-bound version of relation (2.3). By taking $p=1$ in the derivation of (5.4), we obtain $\mathbb{E} \int_{0}^{t} \mathrm{e}^{-\xi_{s}} \mathrm{~d} s=\int_{0}^{t} \mathbb{E} \mathrm{e}^{-\xi_{s}} \mathrm{~d} s<\infty$. Recall that $\mathbb{J}_{F}^{+}<p \leq \kappa$ and $\mathbb{E} \vartheta_{n}^{p}(t)<\infty$ for any $n \geq 1$. Let $N$ be specified as in (5.5)-(5.6). Then, by (5.15), we can take some positive constant $A$ such that, for all $1 \leq n \leq N$,

$$
\mathbb{E}\left(\vartheta_{n}^{p}(t) \mathbf{1}_{\left[D_{0, t}>A\right]}\right)<\frac{\varepsilon}{N^{p+1}}, \quad \mathbb{P}\left(D_{0, t}>A\right)<\frac{\varepsilon}{N},
$$

and

$$
\mathbb{E}\left(\vartheta_{n}^{p}(t) \mathbf{1}_{\left[\int_{0}^{t} \mathrm{e}^{-\xi_{s}} \mathrm{~d} s>A\right]}\right)<\frac{\varepsilon}{N^{p+1}}, \quad \mathbb{P}\left(\int_{0}^{t} \mathrm{e}^{-\xi_{s}} \mathrm{~d} s>A\right)<\frac{\varepsilon}{N}
$$

For the fixed $A$, according to (5.1), we can derive

$$
\begin{aligned}
\Psi(x, t) \geq & \mathbb{P}\left(\sum_{n=1}^{\infty} X_{n} \vartheta_{n}(t)>x+c \int_{0}^{t} \mathrm{e}^{-\xi_{s}} \mathrm{~d} s+\sigma \int_{0}^{t} \mathrm{e}^{-\xi_{s}} \mathrm{~d} B_{s}\right) \\
\geq & \mathbb{P}\left(\sum_{n=1}^{N} X_{n} \vartheta_{n}(t)>x+(c+\sigma) A, D_{0, t} \leq A, \int_{0}^{t} \mathrm{e}^{-\xi_{s}} \mathrm{~d} s \leq A\right) \\
\geq & \mathbb{P}\left(\sum_{n=1}^{N} X_{n} \vartheta_{n}(t)>x+(c+\sigma) A\right)-\mathbb{P}\left(\sum_{n=1}^{N} X_{n} \vartheta_{n}(t)>x, D_{0, t}>A\right) \\
& -\mathbb{P}\left(\sum_{n=1}^{N} X_{n} \vartheta_{n}(t)>x, \int_{0}^{t} \mathrm{e}^{-\xi_{s}} \mathrm{~d} s>A\right) \\
= & : I_{1}^{\prime}(t)-I_{2}^{\prime}(t)-I_{3}^{\prime}(t) .
\end{aligned}
$$

For $I_{1}^{\prime}(t)$, with $L>0$ given in (5.9), it follows from Lemma 4.3 that there exists some $x_{7}>x_{6}$ 
such that, for all $x>x_{7}$,

$$
\begin{aligned}
I_{1}^{\prime}(t) \geq & \mathbb{P}\left(\sum_{n=1}^{N} X_{n} \vartheta_{n}(t)>x+(c+\sigma) A, \bigcap_{j=1}^{N}\left(\vartheta_{j}(t) \leq L\right)\right) \\
\geq & (1-\varepsilon) \sum_{n=1}^{N} \mathbb{P}\left(X_{n} \vartheta_{n}(t)>x+(c+\sigma) A, \bigcap_{j=1}^{N}\left(\vartheta_{j}(t) \leq L\right)\right) \\
= & (1-\varepsilon)\left(\sum_{n=1}^{N} \mathbb{P}\left(X_{n} \vartheta_{n}(t)>x+(c+\sigma) A\right)\right. \\
& \left.\quad-\sum_{n=1}^{N} \mathbb{P}\left(X_{n} \vartheta_{n}(t)>x+(c+\sigma) A, \bigcup_{j=1}^{N}\left(\vartheta_{j}(t)>L\right)\right)\right) \\
= & :(1-\varepsilon)\left[I_{11}^{\prime}(t)-I_{12}^{\prime}(t)\right] .
\end{aligned}
$$

Recall that $X_{n} \vartheta_{n}(t) \in \mathcal{C}$ for any $n \geq 1$. Then, by (4.1), we can take some $x_{8}>x_{7}$ such that, for all $x>x_{8}$,

$$
I_{11}^{\prime}(t) \geq(1-\varepsilon) \sum_{n=1}^{N} \mathbb{P}\left(X_{n} \vartheta_{n}(t)>x\right) .
$$

By the same approach as $I_{12}(t)$, we can show that there exists some $x_{9}>x_{8}$ such that, for all $x>x_{9}$,

$$
I_{12}^{\prime}(t) \leq \sum_{n=1}^{N} \mathbb{P}\left(X_{n} \vartheta_{n}(t)>x, \bigcup_{j=1}^{N}\left(\vartheta_{j}(t)>L\right)\right) \leq C \varepsilon \bar{F}(x) .
$$

Therefore, for all $x>x_{9}$,

$$
I_{1}^{\prime}(t) \geq(1-C \varepsilon) \sum_{n=1}^{N} \mathbb{P}\left(X_{n} \vartheta_{n}(t)>x\right)-C \varepsilon \bar{F}(x) .
$$

For $I_{2}^{\prime}(t)$, by Lemma 4.1(i) and (5.19), we can show that there exists some $x_{10}>x_{9}$ such that, for all $x>x_{10}$,

$$
\begin{aligned}
I_{2}^{\prime}(t) & \leq \mathbb{P}\left(\sum_{n=1}^{N} X_{n} \vartheta_{n}(t)>x, D_{0, t}>A\right) \\
& \leq \sum_{n=1}^{N} \mathbb{P}\left(X_{n} \vartheta_{n}(t)>\frac{x}{N}, D_{0, t}>A\right) \\
& =\sum_{n=1}^{N} \mathbb{E}\left(\mathbf{1}_{\left[D_{0, t}>A\right]} \mathbb{P}\left(X_{n} \vartheta_{n}(t)>\frac{x}{N} \mid \vartheta_{n}(t), D_{0, t}\right)\right) \\
& \leq C \bar{F}(x) \sum_{n=1}^{N}\left[N^{p} \mathbb{E}\left(\vartheta_{n}^{p}(t) \mathbf{1}_{\left[D_{0, t}>A\right]}\right)+\mathbb{P}\left(D_{0, t}>A\right)\right] \\
& \leq C \varepsilon \bar{F}(x) .
\end{aligned}
$$


For $I_{3}^{\prime}(t)$, by Lemma 4.1(i) and (5.20), we can show that there exists some $x_{11}>x_{10}$ such that, for all $x>x_{11}$,

$$
\begin{aligned}
I_{3}^{\prime}(t) & \leq \mathbb{P}\left(\sum_{n=1}^{N} X_{n} \vartheta_{n}(t)>x, \int_{0}^{t} \mathrm{e}^{-\xi_{s}} \mathrm{~d} s>A\right) \\
& \leq \sum_{n=1}^{N} \mathbb{P}\left(X_{n} \vartheta_{n}(t)>\frac{x}{N}, \int_{0}^{t} \mathrm{e}^{-\xi_{s}} \mathrm{~d} s>A\right) \\
& =\sum_{n=1}^{N} \mathbb{E}\left(\mathbf{1}_{\left[\int_{0}^{t} \mathrm{e}^{-\xi_{s}} \mathrm{~d} s>A\right]} \mathbb{P}\left(X_{n} \vartheta_{n}(t)>\frac{x}{N} \mid \vartheta_{n}(t), \int_{0}^{t} \mathrm{e}^{-\xi_{s}} \mathrm{~d} s\right)\right) \\
& \leq C \bar{F}(x) \sum_{n=1}^{N}\left[N^{p} \mathbb{E}\left(\vartheta_{n}^{p}(t) \mathbf{1}_{\left[\int_{0}^{t} \mathrm{e}^{-\xi_{s}} \mathrm{~d} s>A\right]}\right)+\mathbb{P}\left(\int_{0}^{t} \mathrm{e}^{-\xi_{s}} \mathrm{~d} s>A\right)\right] \\
& \leq C \varepsilon \bar{F}(x) .
\end{aligned}
$$

By Lemma 4.1(i) and (5.5), we can take some $x_{12}>x_{11}$ such that, for all $x>x_{12}$,

$$
\begin{aligned}
\sum_{n=N+1}^{\infty} \mathbb{P}\left(X_{n} \vartheta_{n}(t)>x\right) & =\sum_{n=N+1}^{\infty} \mathbb{E}\left(\mathbb{P}\left(X_{n} \vartheta_{n}(t)>x \mid \vartheta_{n}(t)\right)\right) \\
& \leq C \bar{F}(x) \sum_{n=N+1}^{\infty} \mathbb{E}\left(\vartheta_{n}^{p}(t)+\mathbf{1}_{\left[\vartheta_{n}(t) \leq 1\right]}\right) \\
& \leq C \varepsilon \bar{F}(x) .
\end{aligned}
$$

Substituting (5.22)-(5.26) into (5.21) and using (5.17) and (5.27), we obtain, for all $x>x_{12}$,

$$
\begin{aligned}
\Psi(x, t) & \geq(1-C \varepsilon) \sum_{n=1}^{N} \mathbb{P}\left(X_{n} \vartheta_{n}(t)>x\right)-C \varepsilon \bar{F}(x) \\
& \geq(1-C \varepsilon) \sum_{n=1}^{\infty} \mathbb{P}\left(X_{n} \vartheta_{n}(t)>x\right)-C \varepsilon \bar{F}(x) \\
& \geq(1-C \varepsilon) \sum_{n=1}^{\infty} \mathbb{P}\left(X_{n} \vartheta_{n}(t)>x\right) \\
& =(1-C \varepsilon) \lambda \int_{0}^{t} \mathbb{P}\left(X_{1} \mathrm{e}^{-\xi_{s}}>x\right) \mathrm{d} s .
\end{aligned}
$$

Thus, inequalities (5.18) and (5.28) hold for all $x>x_{12}$. By the arbitrariness of $\varepsilon>0$, we obtain

$$
\Psi(x, t) \sim \lambda \int_{0}^{t} \mathbb{P}\left(X_{1} \mathrm{e}^{-\xi_{s}}>x\right) \mathrm{d} s .
$$

It remains to prove relation (2.4) when $F \in \mathcal{R}_{-\alpha}$. Recall that $\mathbb{E}_{n}^{\kappa}(t)<\infty$ for any $n \geq 1$. Then, by Proposition 3 of [3], we have

$$
\mathbb{P}\left(X_{n} \vartheta_{n}(t)>x\right) \sim \bar{F}(x) \mathbb{E} \vartheta_{n}^{\alpha}(t), \quad n \geq 1 .
$$


Note that (5.4) still holds when $p=\alpha$. Then, for the fixed $\varepsilon>0$ as above, we can take some positive integer, also denoted by $N$, such that

$$
\sum_{n=N+1}^{\infty} \mathbb{E} \vartheta_{n}^{\alpha}(t)<\varepsilon .
$$

On the one hand, by (5.18), (5.30), and (5.3), there exists some $x_{1}^{\prime}>x_{6}$ such that, for all $x>x_{1}^{\prime}$,

$$
\begin{aligned}
\Psi(x, t) & \leq(1+C \varepsilon) \sum_{n=1}^{N} \mathbb{P}\left(X_{n} \vartheta_{n}(t)>x\right)+C \varepsilon \mathbb{P}\left(X_{1} \vartheta_{1}(t)>x\right) \\
& \leq(1+C \varepsilon) \bar{F}(x) \sum_{n=1}^{N} \mathbb{E} \vartheta_{n}^{\alpha}(t)+C \varepsilon \bar{F}(x) \mathbb{E} \vartheta_{1}^{\alpha}(t) \\
& \leq(1+C \varepsilon) \bar{F}(x) \sum_{n=1}^{\infty} \mathbb{E} \vartheta_{n}^{\alpha}(t) \\
& =(1+C \varepsilon) \bar{F}(x) \sum_{n=1}^{\infty} \mathbb{E}\left(\mathrm{e}^{-\alpha \xi_{\tau_{n}-}} \mathbf{1}_{\left[\tau_{n} \leq t\right]}\right) \\
& =(1+C \varepsilon) \bar{F}(x) \sum_{n=1}^{\infty} \int_{0}^{t} \mathbb{E}^{-\alpha \xi_{s}} \frac{s^{n-1} \lambda^{n}}{(n-1) !} \mathrm{d} s \\
& =(1+C \varepsilon) \lambda \bar{F}(x) \int_{0}^{t} \mathbb{E} \mathrm{e}^{-\alpha \xi_{s}} \mathrm{~d} s .
\end{aligned}
$$

On the other hand, according to (5.28) and (5.30)-(5.31), there exists some $x_{2}^{\prime}>x_{12}$ such that, for all $x>x_{2}^{\prime}$,

$$
\begin{aligned}
\Psi(x, t) & \geq(1-C \varepsilon) \sum_{n=1}^{N} \mathbb{P}\left(X_{n} \vartheta_{n}(t)>x\right)-C \varepsilon \bar{F}(x) \\
& \geq(1-C \varepsilon) \bar{F}(x) \sum_{n=1}^{N} \mathbb{E} \vartheta_{n}^{\alpha}(t)-C \varepsilon \bar{F}(x) \\
& \geq(1-C \varepsilon) \bar{F}(x) \sum_{n=1}^{\infty} \mathbb{E} \vartheta_{n}^{\alpha}(t)-C \varepsilon \bar{F}(x) \\
& \geq(1-C \varepsilon) \bar{F}(x) \sum_{n=1}^{\infty} \mathbb{E} \vartheta_{n}^{\alpha}(t) \\
& =(1-C \varepsilon) \lambda \bar{F}(x) \int_{0}^{t} \mathbb{E} \mathrm{e}^{-\alpha \xi_{s}} \mathrm{~d} s .
\end{aligned}
$$

Thus, inequalities (5.32) and (5.33) hold for all $x>\max \left\{x_{1}^{\prime}, x_{2}^{\prime}\right\}$. By the arbitrariness of $\varepsilon>0$, we obtain

$$
\Psi(x, t) \sim \lambda \bar{F}(x) \int_{0}^{t} \mathbb{E} \mathrm{e}^{-\alpha \xi_{s}} \mathrm{~d} s .
$$

This completes the proof. 
Proof of Theorem 2.2. We first prove the upper-bound version of relation (2.5). Define $\vartheta_{n}=\mathrm{e}^{-\xi_{\tau_{n}-}}$, and let $\delta_{2}>0$ be an arbitrarily fixed constant. From $\kappa>\max \left\{\mathbb{J}_{F}^{+}, 2\right\}$ and $\eta>\kappa-1+2 \kappa / \mathbb{J}_{F}^{-}$, we see that $\eta>\kappa+1$ and there exists some $p_{1} \in\left(0, \mathbb{J}_{F}^{-}\right)$such that $\eta>\kappa-1+2 \kappa / p_{1}$. Then, by Hölder's inequality and (5.2)-(5.3), we derive, for $p=\kappa$ or $p_{1}$,

$$
\begin{aligned}
& \sum_{n=1}^{\infty} n^{p} \log { }^{p\left(1+\delta_{2}\right)} n \mathbb{E} \vartheta_{n}^{p} \\
& \leq C \sum_{n=1}^{\infty} n^{[p]+1} \mathbb{E}^{-p \xi_{\tau_{n}-}} \\
& =C \sum_{n=1}^{\infty} n^{[p]+1} \int_{0}^{\infty} \mathbb{E} \mathrm{e}^{-p \xi_{s}} \frac{s^{n-1} \lambda^{n}}{(n-1) !} \mathrm{e}^{-\lambda s} \mathrm{~d} s \\
& \leq C \int_{0}^{\infty} \max \left\{s^{[p]+1}, 1\right\} \mathbb{E} \mathrm{e}^{-p \xi_{s}} \mathrm{~d} s \\
& \leq C \int_{0}^{\infty} \max \left\{s^{[p]+1}, 1\right\} \mathbb{E}^{p / \kappa} \mathrm{e}^{-\kappa \xi_{s}} \mathrm{~d} s \\
& =C \int_{0}^{2} \max \left\{s^{[p]+1}, 1\right\} \mathbb{E}^{p / \kappa} \mathrm{e}^{-\kappa \xi_{s}} \mathrm{~d} s+C \int_{2}^{\infty} \frac{s^{[p]+2-p / \kappa} \log ^{1+\delta_{2}} s}{s^{1-p / \kappa} \log ^{1+\delta_{2}} s} \mathbb{E}^{p / \kappa} \mathrm{e}^{-\kappa \xi_{s}} \mathrm{~d} s \\
& \leq C\left(\int_{0}^{2} \mathbb{E}^{-\kappa \xi_{s}} \mathrm{~d} s\right)^{p / \kappa}+C\left(\int_{2}^{\infty} s^{\kappa-1+2 \kappa / p} \log ^{\left(1+\delta_{2}\right) \kappa / p} s \mathbb{E} \mathrm{e}^{-\kappa \xi_{s}} \mathrm{~d} s\right)^{p / \kappa} \\
& \leq C\left(\int_{0}^{2} \mathbb{E} \mathrm{e}^{-\kappa \xi_{s}} \mathrm{~d} s\right)^{p / \kappa}+C\left(\int_{2}^{\infty} s^{\eta} \mathbb{E} \mathrm{e}^{-\kappa \xi_{s}} \mathrm{~d} s\right)^{p / \kappa} \\
& <\infty \text {. }
\end{aligned}
$$

Hence, for arbitrarily fixed $\varepsilon>0$, we can take some integer $N>1$ such that

$$
\begin{gathered}
\sum_{n=N+1}^{\infty}\left(n^{p_{1}} \log ^{p_{1}\left(1+\delta_{2}\right)} n \mathbb{E} \vartheta_{n}^{p_{1}}+n^{\kappa} \log ^{\kappa\left(1+\delta_{2}\right)} n \mathbb{E} \vartheta_{n}^{\kappa}\right)<\varepsilon, \\
\sum_{n=N+1}^{\infty} \frac{1}{n \log ^{1+\delta_{2}} n}<1 .
\end{gathered}
$$

Note that (5.34) implies that $\mathbb{E} \vartheta_{n}^{\kappa}<\infty$ for any $n \geq 1$. Then, according to Lemma 4.1(iii), $X_{n} \vartheta_{n} \in \mathcal{C}$ for any $1 \leq n \leq N$. Hence, by the definition of the class $\mathcal{C}$ in (2.1), we can show that there exist $v_{0} \in\left(\frac{1}{2}, 1\right)$ and $x_{1}>0$ such that, for all $x>x_{1}$,

$$
\bar{F}\left(v_{0} x\right) \leq(1+\varepsilon) \bar{F}(x) \text { and } \sum_{n=1}^{N} \mathbb{P}\left(X_{n} \vartheta_{n}>v_{0} x\right) \leq(1+\varepsilon) \sum_{n=1}^{N} \mathbb{P}\left(X_{n} \vartheta_{n}>x\right)
$$

Again, by (5.34), we have $\mathbb{E}\left(\vartheta_{n}^{p_{1}}+\vartheta_{n}^{\kappa}\right)<\infty$ for any $n \geq 1$. Take $L>0$ such that, for all $1 \leq j, n \leq N$,

$$
\mathbb{E}\left(\vartheta_{n}^{p_{1}} \mathbf{1}_{\left[\vartheta_{j}>L\right]}\right)<\frac{\varepsilon}{N^{p_{1}+2}(1+\varepsilon)}, \quad \mathbb{E}\left(\vartheta_{n}^{\kappa} \mathbf{1}_{\left[\vartheta_{j}>L\right]}\right)<\frac{\varepsilon}{N^{\kappa+2}(1+\varepsilon)} .
$$


Define $D_{0, \infty}=\sup _{0 \leq s<\infty}\left|\int_{0}^{s} \mathrm{e}^{-\xi_{v}} \mathrm{~d} B_{v}\right|$. Note that $\eta>\kappa-1+2 \kappa / \mathbb{J}_{F}^{-}>\kappa / 2-1>0$. Then, by the Burkholder-Davis-Gundy inequalities, Minkowski's inequality, and Hölder's inequality, we can derive

$$
\begin{aligned}
& \mathbb{E} D_{0, \infty}^{\kappa} \leq C \mathbb{E}\left(\int_{0}^{2} \mathrm{e}^{-2 \xi_{s}} \mathrm{~d} s+\int_{2}^{\infty} \mathrm{e}^{-2 \xi_{s}} \mathrm{~d} s\right)^{\kappa / 2} \\
& \leq C\left\{\mathbb{E}^{2 / \kappa}\left(\int_{0}^{2} \mathrm{e}^{-2 \xi_{s}} \mathrm{~d} s\right)^{\kappa / 2}\right.
\end{aligned}
$$

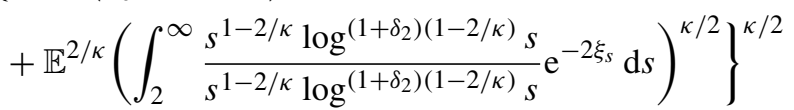

$$
\begin{aligned}
& \leq C\left\{\mathbb{E}^{2 / \kappa} \int_{0}^{2} \mathrm{e}^{-\kappa \xi_{s}} \mathrm{~d} s+\mathbb{E}^{2 / \kappa} \int_{2}^{\infty} s^{\kappa / 2-1} \log \left(1+\delta_{2}\right)(\kappa / 2-1) s \mathrm{e}^{-\kappa \xi_{s}} \mathrm{~d} s\right\}^{\kappa / 2} \\
& \leq C\left\{\left(\int_{0}^{2} \mathbb{E} \mathrm{e}^{-\kappa \xi_{s}} \mathrm{~d} s\right)^{2 / \kappa}+\left(\int_{2}^{\infty} s^{\eta} \mathbb{E} \mathrm{e}^{-\kappa \xi_{s}} \mathrm{~d} s\right)^{2 / \kappa}\right\}^{\kappa / 2} \\
& <\infty \text {. }
\end{aligned}
$$

Recall that $\mathbb{E} \vartheta_{1}^{\kappa}<\infty$. By Lemma 4.1(iii), we can take $x_{2}>x_{1}$ and $C>0$ such that, for all $x>x_{2}$,

$$
\bar{F}(x) \leq C \mathbb{P}\left(X_{1} \vartheta_{1}>x\right)
$$

Now, similarly to the derivations in (5.8)-(5.18) but using Lemma 4.1(ii), (5.35)-(5.37), (5.38), (5.39), and (5.40) instead of Lemma 4.1(i), (5.5)-(5.7), (5.9), (5.15), and (5.17), respectively, it follows from (5.3) that there exists some $x_{3}>x_{2}$ such that, for all $x>x_{3}$,

$$
\begin{aligned}
\Psi(x) & \leq(1+C \varepsilon) \sum_{n=1}^{N} \mathbb{P}\left(X_{n} \vartheta_{n}>x\right)+C \varepsilon \mathbb{P}\left(X_{1} \vartheta_{1}>x\right) \\
& \leq(1+C \varepsilon) \sum_{n=1}^{\infty} \mathbb{P}\left(X_{n} \vartheta_{n}>x\right) \\
& =(1+C \varepsilon) \sum_{n=1}^{\infty} \mathbb{P}\left(X_{n} \mathrm{e}^{\left.-\xi_{\tau_{n}-}>x\right)}\right. \\
& =(1+C \varepsilon) \sum_{n=1}^{\infty} \int_{0}^{\infty} \mathbb{P}\left(X_{1} \mathrm{e}^{-\xi_{s}}>x\right) \frac{s^{n-1} \lambda^{n}}{(n-1) !} \mathrm{e}^{-\lambda s} \mathrm{~d} s \\
& =(1+C \varepsilon) \lambda \int_{0}^{\infty} \mathbb{P}\left(X_{1} \mathrm{e}^{-\xi_{s}}>x\right) \mathrm{d} s .
\end{aligned}
$$

We next prove the lower-bound version of relation (2.5). Recall that $0<p_{1}<\mathbb{J}_{F}^{-}$and $\eta>\kappa-1+2 \kappa / p_{1}$. Take $T_{0}>1$ such that

$$
\int_{T_{0}}^{\infty} \mathbb{E} \mathrm{e}^{-\kappa \xi_{s}} \mathrm{~d} s \leq \int_{T_{0}}^{\infty} s^{\eta} \mathbb{E} \mathrm{e}^{-\kappa \xi_{s}} \mathrm{~d} s<\varepsilon^{\kappa / p_{1}}
$$

Trivially, $\eta>\kappa-1+2 \kappa / p_{1}>\kappa / p_{1}-1$. Then, for the fixed $T_{0}$, by Hölder's inequality, 
we obtain

$$
\begin{aligned}
& \int_{T_{0}}^{\infty} \mathbb{E} \mathrm{e}^{-p_{1} \xi_{s}} \mathrm{~d} s \leq \int_{T_{0}}^{\infty} \mathbb{E}^{p_{1} / \kappa} \mathrm{e}^{-\kappa \xi_{s}} \mathrm{~d} s
\end{aligned}
$$

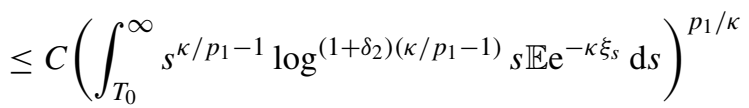

$$
\begin{aligned}
& \leq C\left(\int_{T_{0}}^{\infty} s^{\eta} \mathbb{E} \mathrm{e}^{-\kappa \xi_{s}} \mathrm{~d} s\right)^{p_{1} / \kappa} \\
& <C \varepsilon \text {. }
\end{aligned}
$$

By Lemma 4.1(ii), (5.40), and (5.42)-(5.43), there exists some $x_{4}>x_{3}$ such that, for all $x>x_{4}$,

$$
\begin{aligned}
\int_{T_{0}}^{\infty} \mathbb{P}\left(X_{1} \mathrm{e}^{-\xi_{s}}>x\right) \mathrm{d} s & \leq C \bar{F}(x) \int_{T_{0}}^{\infty}\left\{\mathrm{E}^{-p_{1} \xi_{s}}+\mathbb{E}^{-\kappa \xi_{s}}\right\} \mathrm{d} s \\
& \leq C \varepsilon \bar{F}(x) \\
& \leq C \varepsilon \mathbb{P}\left(X_{1} \vartheta_{1}>x\right) \\
& \leq C \varepsilon \sum_{n=1}^{\infty} \mathbb{P}\left(X_{n} \vartheta_{n}>x\right) \\
& =C \varepsilon \lambda \int_{0}^{\infty} \mathbb{P}\left(X_{1} \mathrm{e}^{-\xi_{s}}>x\right) \mathrm{d} s
\end{aligned}
$$

By relation (5.29) with $t$ replaced by $T_{0}$, we have, for all $x>x_{4}$,

$$
\begin{aligned}
\Psi(x) \geq \Psi\left(x, T_{0}\right) & \sim \lambda \int_{0}^{T_{0}} \mathbb{P}\left(X_{1} \mathrm{e}^{-\xi_{s}}>x\right) \mathrm{d} s \\
& =\lambda\left(\int_{0}^{\infty}-\int_{T_{0}}^{\infty}\right) \mathbb{P}\left(X_{1} \mathrm{e}^{-\xi_{s}}>x\right) \mathrm{d} s \\
& \geq(1-C \varepsilon) \lambda \int_{0}^{\infty} \mathbb{P}\left(X_{1} \mathrm{e}^{-\xi_{s}}>x\right) \mathrm{d} s .
\end{aligned}
$$

Thus, inequalities (5.41) and (5.44) hold for all $x>x_{4}$. By the arbitrariness of $\varepsilon$, we obtain

$$
\Psi(x) \sim \lambda \int_{0}^{\infty} \mathbb{P}\left(X_{1} \mathrm{e}^{-\xi_{s}}>x\right) \mathrm{d} s .
$$

It remains to prove relation (2.6) when $F \in \mathcal{R}_{-\alpha}$. Recall that $\mathbb{E} \vartheta_{n}^{\kappa}<\infty$ for any $n \geq 1$. Then, by Proposition 3 of [3], we have

$$
\mathbb{P}\left(X_{n} \vartheta_{n}>x\right) \sim \bar{F}(x) \mathbb{E} \vartheta_{n}^{\alpha}, \quad n \geq 1 .
$$

Note that (5.34) still holds when $p=\alpha$ since $\eta>\kappa-1+2 \kappa / \alpha$. Hence, for the fixed $\varepsilon$ as above, we can take some positive integer, also denoted by $N$, such that

$$
\sum_{n=N+1}^{\infty} \mathbb{E} \vartheta_{n}^{\alpha}<\varepsilon
$$


On the one hand, similarly to the derivation in (5.32) but using (5.45)-(5.46) instead of (5.30)(5.31), respectively, we can show that there exists some $x_{5}>x_{4}$ such that, for all $x>x_{5}$,

$$
\Psi(x) \leq(1+C \varepsilon) \lambda \bar{F}(x) \int_{0}^{\infty} \mathbb{E} \mathrm{e}^{-\alpha \xi_{s}} \mathrm{~d} s .
$$

On the other hand, by (5.44)-(5.46), we can show that there exists some $x_{6}>x_{5}$ such that, for all $x>x_{6}$,

$$
\begin{aligned}
\Psi(x) & \geq(1-C \varepsilon) \lambda \int_{0}^{\infty} \mathbb{P}\left(X_{1} \mathrm{e}^{-\xi_{s}}>x\right) \mathrm{d} s \\
& =(1-C \varepsilon) \sum_{n=1}^{\infty} \mathbb{P}\left(X_{n} \vartheta_{n}>x\right) \\
& \geq(1-C \varepsilon) \sum_{n=1}^{N} \mathbb{P}\left(X_{n} \vartheta_{n}>x\right) \\
& \geq(1-C \varepsilon) \bar{F}(x) \sum_{n=1}^{N} \mathbb{E} \vartheta_{n}^{\alpha} \\
& \geq(1-C \varepsilon) \bar{F}(x) \sum_{n=1}^{\infty} \mathbb{E} \vartheta_{n}^{\alpha} \\
& =(1-C \varepsilon) \bar{F}(x) \lambda \int_{0}^{\infty} \mathbb{E} \mathrm{e}^{-\alpha \xi_{s}} \mathrm{~d} s .
\end{aligned}
$$

Hence, inequalities (5.47) and (5.48) hold for all $x>x_{6}$. By the arbitrariness of $\varepsilon$, we obtain (2.6).

\section{Acknowledgements}

The authors would like to thank the anonymous referee for his/her valuable comments which greatly improved the presentation of the paper. The first author was supported by the National Natural Science Foundation of China (grant numbers 71271042 and 71001017). The second author was supported by the National Natural Science Foundation of China (grant number 71271042), the plan of Jiangsu Specially-Appointed Professors, Jiangsu Hi-level Innovative and Entrepreneurship Talent Introduction Plan, and the Major Program of Key Research Center in Financial Risk Management of Jiangsu Universities Philosophy Social Sciences (grant number 2012JDXM009).

\section{References}

[1] Biagini, F., Hu, Y., ØKsendal, B. And Zhang, T. (2008). Stochastic Calculus for Fractional Brownian Motion and Applications. Springer, London.

[2] Bingham, N. H., Goldie, C. M. And Teugels, J. L. (1987). Regular Variation. Cambridge University Press.

[3] Breiman, L. (1965). On some limit theorems similar to the arc-sin law. Theoret. Prob. Appl. 10, $323-331$.

[4] Brigo, D. ANd Mercurio, F. (2001). Interest Rate Models-Theory and Practice. Springer, Berlin.

[5] CAI, J. AND TANG, Q. (2004). On max-sum equivalence and convolution closure of heavy-tailed distributions and their applications. J. Appl. Prob. 41, 117-130.

[6] CAI, J. AND YANG, H. (2005). Ruin in the perturbed compound Poisson risk process under interest force. Adv. Appl. Prob. 37, 819-835.

[7] Chen, Y. AND NG, K. W. (2007). The ruin probability of the renewal model with constant interest force and negatively dependent heavy-tailed claims. Insurance Math. Econom. 40, 415-423. 
[8] Cline, D. B. H. and Samorodnitsky, G. (1994). Subexponentiality of the product of independent random variables. Stoch. Process. Appl. 49, 75-98.

[9] Collamore, J. F. (2009). Random recurrence equations and ruin in a Markov-dependent stochastic economic environment. Ann. Appl. Prob. 19, 1404-1458.

[10] Drăgulescu, A. A. And Yakovenko, V. M. (2002). Probability distribution of returns in the Heston model with stochastic volatility. Quant. Finance 2, 443-453.

[11] Dufresne, D. (2001). The integrated square-root process. Research paper.

[12] Emmer, S. AND KlÜPpelberg, C. (2004). Optimal portfolios when stock prices follow an exponential Lévy process. Finance Stoch. 8, 17-44.

[13] Frolova, A., Kabanov, Y. and Pergamenshchikov, S. (2002). In the insurance business risky investments are dangerous. Finance Stoch. 6, 227-235.

[14] Gaier, J. And Grandits, P. (2002). Ruin probabilities in the presence of regularly varying tails and optimal investment. Insurance Math. Econom. 30, 211-217.

[15] Goldie, C. M. (1991). Implicit renewal theory and tails of solutions of random equations. Ann. Appl. Prob. 1, 126-166.

[16] Heyde, C. C. AND WANG, D. (2009). Finite-time ruin probability with an exponential Lévy process investment return and heavy-tailed claims. Adv. Appl. Prob. 41, 206-224.

[17] Kalashnikov, V. and Norberg, R. (2002). Power tailed ruin probabilities in the presence of risky investments. Stoch. Process. Appl. 98, 211-228.

[18] KlüPpelberg, C. And Kostadinova, R. (2008). Integrated insurance risk models with exponential Lévy investment. Insurance Math. Econom. 42, 560-577.

[19] Konstantinides, D. G. And Miкosch, T. (2005). Large deviations and ruin probabilities for solutions to stochastic recurrence equations with heavy-tailed innovations. Ann. Prob. 33, 1992-2035.

[20] Mikosch, T. and Samorodnitsky, G. (2000). The supremum of a negative drift random walk with dependent heavy-tailed steps. Ann. Appl. Prob. 10, 1025-1064.

[21] Nelsen, R. B. (2006). An Introduction to Copulas, 2nd edn. Springer, New York.

[22] PAulsen, J. (2002). On Cramér-like asymptotics for risk processes with stochastic return on investments. Ann. Appl. Prob. 12, 1247-1260.

[23] Paulsen, J. and Gjessing, H. K. (1997). Ruin theory with stochastic return on investments. Adv. Appl. Prob. 29, 965-985.

[24] Protter, P. (2004). Stochastic Integration and Differential Equations, 2nd edn. Springer, Berlin.

[25] Shreve, S. E. (2004). Stochastic Calculus for Finance II. Springer, New York.

[26] TANG, Q. (2006). Insensitivity to negative dependence of the asymptotic behavior of precise large deviations. Electron. J. Prob. 11, 107-120.

[27] WANG, D. AND TANG, Q. (2004). Maxima of sums and random sums for negatively associated random variables with heavy tails. Stat. Prob. Lett. 68, 287-295.

[28] WANG, D. AND TANG, Q. (2006). Tail probabilities of randomly weighted sums of random variables with dominated variation. Stoch. Models 22, 253-272.

[29] Wang, D., Chen, P. And Su, C. (2007). The supremum of random walk with negatively associated and heavytailed steps. Statist. Prob. Lett. 77, 1403-1412.

[30] Wang, D., Su, C. ANd Zeng, Y. (2005). Uniform estimate for maximum of randomly weighted sums with applications to insurance risk theory. Sci. China Ser. A 48, 1379-1394.

[31] Zhang, Y., Shen, X. And Weng, C. (2009). Approximation of the tail probability of randomly weighted sums and applications. Stoch. Process. Appl. 119, 655-675. 